\title{
Topological finite-determinacy of functions with non-isolated singularities
}

Javier Fernández de Bobadilla

\begin{abstract}
We introduce the concept of topological finite-determinacy for germs of analytic functions within a fixed ideal $I$, which provides a notion of topological finite-determinacy of functions with non-isolated singularities. We prove the following statement which generalizes classical results of Thom and Varchenko: let $A$ be the complement in the ideal $I$ of the space of germs whose topological type remains unchanged under a deformation within the ideal that only modifies sufficiently large order terms of the Taylor expansion. Then $A$ has infinite codimension in $I$ in a suitable sense. We also prove the existence of generic topological types of families of germs of $I$ parametrized by an irreducible analytic set.
\end{abstract}

Mathematics Subject Classification (2000). Primary 32S15, 58K40.

Keywords. Non-isolated singularities, topological finite-determinacy, discriminants.

\section{Introduction}

R. Thom announced in [17] his Stabilisation Theorem stating the following: let $J^{r}(n, m)$ denote the space of $r$-jets of germs at the origin of differentiable mappings from $\mathbb{R}^{n}$ to $\mathbb{R}^{m}$, and $\pi_{r}^{s}: J^{s}(n, m) \rightarrow J^{r}(n, m)$ the natural projection mapping. Consider $f \in J^{r}(n, m)$. There exists a positive integer $s$, depending only on $n, m$ and $r$, and a proper algebraic subset $\Sigma \subset\left(\pi_{r}^{s}\right)^{-1}(f)$ such that any two germs $g_{1}$ and $g_{2}$ with the same $s$-jet belonging to $\left(\pi_{r}^{s}\right)^{-1}(f) \backslash \Sigma$ have the same topological type.

Although R. Thom gave in [17] rather detailed ideas for the proof of his theorem, the first complete proof was given in [18], [19] by A. Varchenko, and followed a completely different approach. More in the line of R. Thom's ideas, E. Looijenga's thesis contains the result in the function case (that is, when $m=1$ ). Later, A. du Plessis (see [11]) gave another proof for arbitrary $m$ based on Thom's suggestions, using also his own ideas and ideas from Mather (actually both A. Varchenko and A. du Plessis gave slightly stronger statements than R. Thom's). In this paper

Supported by the Netherlands Organisation for Scientific Research (NWO). Supported by the Spanish MCyT project BFM2001-1448-C02-01. 
we are interested in a generalization of these results valid in the realm of (complex or real) analytic non-isolated hypersurface singularities. In order to see what kind of properties are desirable let us state A. Varchenko's results in the setting of complex analytic functions: let $J^{r}\left(\mathbb{C}^{n}, \mathbb{C}\right)_{O}$ be the space of $r$-jets of germs of holomorphic functions at the origin $O$ of $\mathbb{C}^{n}$. Denote by $\mathcal{D}$ the group of germs of biholomorphisms fixing the origin of $\mathbb{C}^{n}$. There is a natural action of $\mathcal{D}$ in $J^{r}\left(\mathbb{C}^{n}, \mathbb{C}\right)_{O}$ by composition on the right.

Theorem 1 (Varchenko [18]). Let $T \subset J^{r}\left(\mathbb{C}^{n}, \mathbb{C}\right)_{O}$ be an irreducible algebraic subset. There exists $s \geq r$, and a proper algebraic subset $A \subset\left(\pi_{r}^{s}\right)^{-1}(T)$ such that any two germs $f_{1}$ and $f_{2}$ whose $s$-jet is in $\left(\pi_{r}^{s}\right)^{-1}(T) \backslash A$ have the same topological type.

Moreover, for each $r \geq 1$ there exists a partition of $J^{r}\left(\mathbb{C}^{n}, \mathbb{C}\right)_{O}$ into disjoint constructible subsets $U_{0}^{r}, \ldots, U_{k(r)}^{r}$, invariant by the action of $\mathcal{D}$, such that:

(1) If $i>0$, any two germs $f_{1}$ and $f_{2}$ whose $r$-jet is in $U_{i}^{r}$ has the same topological type.

(2) The codimension of $U_{0}^{r}$ tends to infinity as $r$ increases.

The subsets $U_{i}^{r}$ can be constructed so that, if $s>r$ and $i>0$, then $\left(\pi_{r}^{s}\right)^{-1}\left(U_{i}^{r}\right)$ coincides with one of the subsets $U_{j}^{s}$ with $j>0$. This enables to decompose $\mathcal{O}_{\mathbb{C}^{n}, O}$ as a union of subsets $\left\{V_{i}\right\}_{i \in \mathbb{Z}_{>0}}$ such that for $i>0$, the $V_{i}$ 's are formed by germs of fixed topological type determined by their $r(i)$-jet (for a number $r(i)$ only depending on $i$ ), and $V_{0}$ is infinite codimensional in a suitable sense (and therefore easily avoidable by deformation).

Observe that the topological type of a function $f \in \mathcal{O}_{\mathbb{C}^{n}, O}$ with non-isolated singularities is not determined by any $r$-jet of it, no matter how big is $r$ : summation of a generic homogeneous polynomial of arbitrarily high degree transforms it into a function with an isolated singularity at the origin, whose sheaf of vanishing cycles is concentrated at the origin, unlike the sheaf of vanishing cycles of $f$. Therefore the functions defining non-isolated singularities belong to the residual set $V_{0}$ of the decomposition given above and, consequently, Theorem 1 is only meaningful for the study of isolated singularities.

The object of this paper is to prove a replacement of Theorem 1 which is meaningful for the study of non-isolated singularities of (complex or real) analytic functions. Our strategy is to work with functions belonging to a fixed ideal $I$ instead of the whole space of analytic germs at the origin (for example, if we want to study functions which are singular at a line, we can take $I$ to be the square of the ideal defining it). In this paper we prove a generalization of Theorem 1 valid for any ideal of germs of complex or real analytic functions.

Working within a fixed ideal has been already successful in the study of nonisolated singularities: generalized versality and analytic finite-determinacy, study of the Milnor fibration... (see for example [5], [8], [9], [10], [13], [14], [15], [20]). Many of these papers use a generalized morsification method that consists in de- 
forming non-isolated singularities within a fixed ideal $I$ to get simpler ones, and then study their properties. Up to now this worked only when $I$ has simple geometric properties: it is the square of a complete intersection ideal defining an isolated singularity, or the analytic space defined by it is low dimensional. In order to establish the morsification method in general the generalization Theorem 1 provided in this paper is needed. The generalized Morsification Method appears in [6].

Furthermore, the study of functions within an ideal is also relevant for the study of isolated singularities satisfying a fixed amount of conditions (having some fixed tangencies or multiplicities at infinitely near points...).

Unlike in the case of isolated singularities, the interesting phenomena in the study of functions with non-isolated singularities are not concentrated at the origin, but at a neighbourhood at the origin of the singular locus. This makes insufficient in practice the straightforward generalization of Theorem 1, in which the ring $\mathcal{O}_{\mathbb{C}^{n}, O}$ is replaced by the ideal $I$. We will need a stronger formulation in which we consider, instead of jets at the origin, jets at points ranging in a fixed neighbourhood at the origin: view the ideal $I$ as the stalk at the origin of a coherent ideal sheaf $\tilde{I}$ defined in a neighbourhood $W$ of the origin. Define

$$
J^{r}(W, \tilde{I}):=\coprod_{x \in W} \tilde{I}_{x} / \mathbf{m}_{x}^{r+1} \cap \tilde{I}_{x},
$$

where $\tilde{I}_{x}$ is the stalk of $\tilde{I}$ at $x$, and $\mathbf{m}_{x}$ the ideal of analytic functions vanishing at $x$. For any non-negative integer $r$ we will stratify the set $J^{r}(W, \tilde{I})$ in such a way that each stratum is an analytic variety. The generalization of the subsets $T, A$ and $U_{i}^{r}$ in Theorem 1 will be analytic subsets of the strata of $J^{r}(W, \tilde{I})$ satisfying analogous properties.

As we are working on a neighbourhood $W$ of the origin, the right generalization of the invariance of the $U_{i}^{r}$ 's by the action of $\mathcal{D}$ consists in the following property: the subsets generalizing the $U_{i}^{r}$ 's are invariant by the action of diffeomorphisms between open subsets of $W$ which preserve the ideal sheaf $\tilde{I}$. Actually, what we will prove is the invariance of such subsets by flows within $W$ preserving $\tilde{I}$. This can be viewed as an infinitesimal version of the invariance by diffeomorphisms, and turns out to be enough for applications.

The structure of the paper is the following: first we stratify the spaces $J^{r}(W, \tilde{I})$ for any $r \leq \infty$ and show that the strata are analytic varieties in a natural way when $r<\infty$, and a limit of analytic varieties when $r=\infty$. Later we define the class of closed analytic subsets of the strata of $J^{\infty}(W, \tilde{I})$, their irreducible components and codimension. We also introduce the concept of residual subsets of $J^{\infty}(W, \tilde{I})$, which, roughly speaking, is a subset of infinite codimension. Then we state a proposition that generalizes the main proposition of [18] to our setting. In essence it states that given any irreducible analytic subset of a stratum of $J^{\infty}(W, \tilde{I})$ there is a proper closed analytic subset (discriminant) of it such that two germs in the same connected component of its complement are topologically finite-determined and topologically equivalent. Then, letting residual subsets enter in the picture, we show that such a discriminant is unique, provided that it satisfies a certain 
minimality condition. After we define the concept of flow-invariant subsets of $J^{\infty}(W, \tilde{I})$ as a replacement of the concept of diffeomorphism-invariant subsets of $\mathcal{O}_{\mathbb{C}^{n}, O}$. Then we can formulate the main result of the paper, which generalizes Theorem 1 to our setting, and also shows that the predicted subsets are minimal and unique in a certain sense. In the proof of our main result the invariance of the relevant subsets by flows preserving the ideal sheaf $\tilde{I}$ gets involved, in contrast with the proof of the invariance by the action of $\mathcal{D}$ of Theorem 1, which is straightforward. We leave for the last section the proof of the proposition stating the existence of discriminants. For this we have found A. Varchenko's ideas rather suitable for our setting. Nevertheless, a naive generalization of Varchenko's proof collapses in a fundamental way in several places: summation of generic functions of high order is needed, and this takes us out of our ideal sheaf.

The author would like to thank the referee and Isabel Hernandez Navarro for suggestions concerning the exposition.

\section{The analytic structure of $J^{r}(W, \tilde{I})$}

Let the field $\mathbb{K}$ be either $\mathbb{R}$ or $\mathbb{C}$. Denote by $\mathcal{E}_{W}$ the sheaf of analytic functions on an open subset $W$ of $\mathbb{K}^{n}$ (when $\mathbb{K}=\mathbb{C}$ the sheaf $\mathcal{E}_{W}$ is the sheaf of holomorphic functions $\left.\mathcal{O}_{W}\right)$. For any $x \in W$ denote by $\mathbf{m}_{x}$ the maximal ideal of the stalk of $\mathcal{E}_{W, x}$.

When $\mathbb{K}=\mathbb{R}$ we will work with an special type of real analytic subsets. Consider $\mathbb{R}^{n}$ as the subset of $\mathbb{C}^{n}$ consisting of the points with real coordinates. Let $W \subset \mathbb{R}^{n}$ be an open subset. A $C$-analytic subset of $W$ is a closed real analytic subset $T \subset W$ such that there exists an open neighbourhood $W^{*}$ of $W$ in $\mathbb{C}^{n}$ satisfying $W^{*} \cap \mathbb{R}^{n}=W$ and a closed complex analytic subset $T^{*} \subset W^{*}$ such that $T^{*} \cap \mathbb{R}^{n}=T$. A Zarisky locally closed $C$-analytic subset of $W$ is the difference between two $C$-analytic subsets of $W$. We use Ch. 5 of [12] as a general survey reference for $C$-analytic subsets. Proofs are due to H. Cartan, F. Bruhat and H. Whitney, and can be found in [4], and, mostly, in [3]. For the convenience of the reader we recall the properties that are convenient for us:

A real analytic subset $T \subset W$ is $C$-analytic if and only if there exist a coherent ideal sheaf in $\mathcal{E}_{W}$ whose zero-set is $T$. Any real analytic subset is locally $C$ analytic. Any (possibly infinite) intersection of $C$-analytic subsets is a $C$-analytic subset. Any locally finite union of $C$-analytic subsets is $C$-analytic. The inverse image of a $C$-analytic subset by an analytic mapping is a $C$-analytic subset. A $C$-analytic subset is $C$-irreducible if it is not the union of two $C$-analytic subsets different from itself (a $C$-irreducible $C$-analytic subset needs not be irreducible as a real analytic set). Given a $C$-analytic subset $T \subset W$ there exists a unique irredundant, locally finite family of $C$-irreducible $C$-analytic subsets whose union is $T$. There is a notion of dimension of $C$-analytic subsets which satisfies the following properties: let $T^{\prime} \subset T$ be $C$-analytic subsets of $W$, where $T$ is $C$ - 
irreducible, then $\operatorname{dim}\left(T^{\prime}\right)<\operatorname{dim}(T)$. If $T$ is a $C$-analytic subset of dimension $p$, there is a $C$-analytic subset $T^{\prime} \subset T$ such that $\operatorname{dim}\left(T^{\prime}\right)<p$, and $T \backslash T^{\prime}$ is an analytic manifold of dimension $p$; also, any point of $T$ can be approximated by points where $T$ is an analytic manifold of dimension $p$. A complexification of a real $(C)$-analytic subset $T$ is a complex analytic variety $T^{*}$ such that $T$ is embedded in $T^{*}$ as a real analytic variety, and for each point $t \in T$ there is:

- a neighbourhood $U^{*}$ of $t$ in $T^{*}$,

- a closed analytic subset $Y^{*}$ of an open subset of $\mathbb{C}^{n}$,

- and a complex analytic isomorphism $\varphi: U^{*} \rightarrow Y^{*}$

such that $Y^{*} \cap \mathbb{R}^{n}=\varphi\left(T \cap U^{*}\right)$. Let $T$ be a $C$-analytic subset of $W$ and $T^{*}$ a complexification of $T$. Then $C \subset T$ is a $(C)$-analytic subset of $W$ if and only if there is an open neighbourhood $U^{*}$ of $T$ in $T^{*}$ and a closed complex analytic subset of $C^{*}$ of $U$ such that $C^{*} \cap T=C$.

All the properties above are standard in the complex analytic setting whenever they make sense.

We will adopt the following notational convention: when we work simultaneously with $\mathbb{K}=\mathbb{R}, \mathbb{C}$ and we write $(C)$-analytic, we mean $C$-analytic when $\mathbb{K}=\mathbb{R}$, and complex-analytic when $\mathbb{K}=\mathbb{C}$. When we write just analytic, we mean just real-analytic or complex-analytic depending on whether $\mathbb{K}$ equals $\mathbb{R}$ or $\mathbb{C}$. When we say that a $(C)$-analytic subset is irreducible we mean $C$-irreducible when $\mathbb{K}=\mathbb{R}$, and just irreducible when $\mathbb{K}=\mathbb{C}$.

Let $O$ denote the origin of $\mathbb{K}^{n}$. Consider an ideal $I \subset \mathcal{E}_{O}$; let $\left\{f_{1}, \ldots, f_{m}\right\}$ be a set generators of it. Consider an open neighbourhood $W$ of the origin where each of the generators is defined. Then $\left\{f_{1}, \ldots, f_{m}\right\}$ generate a coherent ideal sheaf $\tilde{I}$ whose stalk $\tilde{I}_{O}$ is equal to $I$.

For any $V \subset W$ we define

$$
J^{r}(V, \tilde{I}):=\coprod_{x \in V} \tilde{I}_{x} / \mathbf{m}_{x}^{r+1} \cap \tilde{I}_{x} \quad J^{\infty}(V, \tilde{I}):=\coprod_{x \in V} \tilde{I}_{x} .
$$

If $0 \leq r \leq s \leq \infty$ there are obvious projection mappings

$$
\pi_{r}^{s}: J^{s}(V, \tilde{I}) \rightarrow J^{r}(V, \tilde{I})
$$

For any $r \leq \infty$ there is another natural projection mapping

$$
p r_{r}: J^{r}(V, \tilde{I}) \rightarrow V
$$

whose fibre $J^{r}(V, \tilde{I})_{x}$ over a point $x \in V$ is, if $r<\infty$, the vector space $\tilde{I}_{x} / \mathbf{m}_{x}^{r+1} \cap \tilde{I}_{x}$, and, if $r=\infty$, the space $\tilde{I}_{x}$.

For any $x \in W$ define the function $\mu_{x}: \mathbb{Z}_{\geq 0} \rightarrow \mathbb{Z}$ by the formula

$$
\mu_{x}(r):=\operatorname{dim}_{\mathbb{K}}\left(\tilde{I}_{x} / \mathbf{m}_{x}^{r+1} \cap \tilde{I}_{x}\right) .
$$

Consider the Hilbert-Samuel function $H_{x}$ of the $\mathcal{E}_{W, x}$-module $\mathcal{M}_{x}:=\mathcal{E}_{W, x} / \tilde{I}_{x}$. As

$$
H_{x}(r)=\operatorname{dim}_{\mathbb{K}}\left(\mathcal{M}_{x} / \mathbf{m}_{x}^{r+1} \mathcal{M}_{x}\right)=\operatorname{dim}_{\mathbb{K}}\left(\frac{\mathcal{E}_{W, x} / \mathbf{m}_{x}^{r+1}}{\tilde{I}_{x}+\mathbf{m}_{x}^{r+1} / \mathbf{m}_{x}^{r+1}}\right)
$$


we deduce that $\mu_{x}(r)=\operatorname{dim}_{\mathbb{K}}\left(\mathcal{E}_{W, x} / \mathbf{m}_{x}^{r+1}\right)-H_{x}(r)$. In [2] the Zarisky analytic upper-semicontinuity of the function $H_{x}$ is proved. Therefore the function $\mu$ is Zarisky analytic lower-semicontinous. As any real-analytic subset is locally $C$ analytic, by shrinking $W$ we can assume that the subset of $W$ where the function $\mu$ is smaller or equal than a given function is a closed $(C)$-analytic subset.

Definition 2. We define the Hilbert-Samuel stratification of $W$ with respect to $\tilde{I}$ to be the minimal partition of $W$ such that $\mu_{x}=\mu_{y}$ for any two points $x$ and $y$ in the same stratum. The strata are Zarisky locally closed $(C)$-analytic subsets, and we will call them the $\tilde{I}$-strata of $W$.

We will use the following notations: consider an analytic function $f$ on an open subset $U \subset \mathbb{K}^{m}$. For any $x \in U$ we denote the germ of $f$ at $x$ by $f_{x}$. For any positive integer $r$ we denote the $r$-jet of $f$ at $x$ by $j^{r} f_{x}$. Denote by $J^{r}\left(M, \mathbb{K}^{m}\right)$ the manifold of $r$-jets of mappings from an analytic manifold $M$ to $\mathbb{K}^{m}$. It has a natural structure of vector bundle over $M$. Given any subset $X$ of $M$, we denote by $J^{r}\left(M, \mathbb{K}^{m}\right)_{\mid X}$ the restriction of the bundle to $X$.

For any positive integer $r$ and any $\tilde{I}$-stratum $X$ of $W$ we endow

$$
p r_{r}: J^{r}(X, \tilde{I}) \rightarrow X
$$

with a natural structure of analytic vector bundle as follows: define the $\mathcal{E}_{W^{-}}$ homomorphism $\varphi: \mathcal{E}_{W}^{m} \rightarrow \mathcal{E}_{W}$ by the formula $\varphi\left(g_{1}, \ldots, g_{m}\right):=\sum_{k=1}^{m} g_{k} f_{k}$. Taking $r$-jets for any positive integer $r$, we obtain a mapping

$$
\varphi^{r}: J^{r}\left(W, \mathbb{K}^{m}\right) \rightarrow J^{r}(W, \mathbb{K}),
$$

given by the formula $\varphi^{r}\left(j^{r} g_{1, x}, \ldots, j^{r} g_{m, x}\right):=\sum_{k=1}^{m} j^{r}\left(g_{k} f_{k}\right)_{x}$. We observe that $\varphi^{r}$ is a homomorphism between trivial analytic vector bundles over $W$, whose image is canonically identified with $J^{r}(W, \tilde{I})$. Then the restriction

$$
\varphi_{\mid X}^{r}: J^{r}\left(W, \mathbb{K}^{k}\right)_{\mid X} \rightarrow J^{r}(W, \mathbb{K})_{\mid X}
$$

is a homomorphism of constant rank between trivial analytic vector bundles. Therefore, its image $J^{r}(X, \tilde{I})$ has a structure of locally trivial analytic vector bundle over $X$ whose rank is $r(X)=\mu_{x}(r)$ (for $x \in X$ arbitrary) and with projection mapping $p r_{r}$. Furthermore, the inclusion $J^{r}(X, \tilde{I}) \hookrightarrow J^{r}(W, \mathbb{K})_{\mid X}$ is a monomorphism of analytic vector bundles.

We denote by $\partial X$ the closed $(C)$-analytic subset of $W$ given by $\bar{X} \backslash X$. It is clear that $J^{r}(X, \tilde{I})$ is closed analytic in $J^{r}(W \backslash \partial X, \mathbb{K})$. Suppose that $\mathbb{K}=\mathbb{R}$. We claim that $J^{r}(X, \tilde{I})$ is actually closed $C$-analytic in $J^{r}(W \backslash \partial X, \mathbb{R})$. View $\mathbb{R}^{n}$ as the set of points of $\mathbb{C}^{n}$ with real coordinates. Locally around any $x \in W$, each generator $f_{i}$ is given by a convergent power series. Therefore, there exists an open subset $W^{*} \subset \mathbb{C}^{n}$ such that $W^{*} \cap \mathbb{R}^{n}=W$, and complex analytic functions $f_{1}^{*}, \ldots, f_{m}^{*}$ defined on $W^{*}$ extending $f_{1}, \ldots, f_{m}$. Let $\tilde{I}^{*}$ be the coherent sheaf generated by them. Given any point $x \in X$, we have

$$
\tilde{I}_{x}^{*} / \mathbf{m}_{x}^{r+1} \cap \tilde{I}_{x}^{*} \cong\left(\tilde{I}_{x} / \mathbf{m}_{x}^{r+1} \cap \tilde{I}_{x}\right) \otimes_{\mathbb{R}} \mathbb{C},
$$


where $\mathbf{m}_{x}$ denotes respectively in each side the maximal ideal of $\mathcal{O}_{W^{*}, x}$ and of $\mathcal{E}_{W, x}$. It follows easily that there is an $\tilde{I}^{*}$-stratum $X^{*}$ of $W^{*}$ satisfying $X^{*} \cap W=$ $X$. Moreover $\partial X^{*} \cap W=\partial X$. The real analytic manifold $J^{r}(W \backslash \partial X, \mathbb{R})$ is naturally embedded as the real part of $J^{r}\left(W^{*} \backslash \partial X^{*}, \mathbb{C}\right)$. As $J^{r}(X, \tilde{I})$ is equal to $J^{r}\left(X^{*}, \tilde{I}^{*}\right) \cap J^{r}(W \backslash \partial X, \mathbb{R})$ and $J^{r}\left(X^{*}, \tilde{I}^{*}\right)$ is closed analytic in $J^{r}\left(W^{*} \backslash \partial X^{*}, \mathbb{C}\right)$, our claim is proved.

A subset $C$ of $J^{\infty}(X, \tilde{I})$ is $r$-determined if it is of the form $C=\left(\pi_{r}^{\infty}\right)^{-1}\left(C^{\prime}\right)$ for a certain subset $C^{\prime}$ of $J^{r}(X, \tilde{I})$. The determinacy degree of a subset $C$ of $J^{\infty}(X, \tilde{I})$ is the minimal integer $r$ such that $C$ is $r$-determined.

Definition 3. A finitely-determined closed $(C)$-analytic subset of $J^{\infty}(X, \tilde{I})$ is a $r$-determined subset for a certain integer $r$, such that $\pi_{r}^{\infty}(C)$ is a closed $(C)$ analytic subset in $J^{r}(X, \tilde{I})$. A finitely-determined locally closed $(C)$-analytic subset is the difference between two finitely-determined closed $(C)$-analytic subsets. The irreducible (connected) components of a $r$-determined (locally) closed $(C)$-analytic subset $C$ are defined to be the inverse images by $\pi_{r}^{\infty}$ of the irreducible (connected) components of $\pi_{r}^{\infty}(C)$.

We endow $J^{\infty}(X, \tilde{I})$ with the final topology for the family of projections $\left\{\pi_{r}^{\infty}\right\}_{r \in \mathbb{N}}$. Then, a family $\left\{C_{j}\right\}_{j \in J}$ of finitely-determined subsets of $J^{\infty}(X, \tilde{I})$ is locally finite if for any $f \in J^{\infty}(X, \tilde{I})$ there exists a positive integer $r$ and a neighbourhood $U$ of $\pi_{r}^{\infty}(f) \in J^{r}(X, \tilde{I})$ such that $\left(\pi_{r}^{\infty}\right)^{-1}(U)$ meets only finitely many $C_{j}$ 's. Choosing $r$ high enough we can assume that each of the subsets that $\left(\pi_{r}^{\infty}\right)^{-1}(U)$ meets are $r$-determined. Therefore the union $\cup_{j \in J} C_{j}$ looks locally like a finite-determined subset. This motivates

Definition 4. A closed $(C)$-analytic subset of $J^{\infty}(X, \tilde{I})$ is the union of a locally finite family of finitely-determined closed $(C)$-analytic subsets of $J^{\infty}(X, \tilde{I})$. A locally closed $(C)$-analytic subset is the difference between two closed $(C)$-analytic subsets. The set of irreducible components of a (locally) closed $(C)$-analytic subset of $J^{\infty}(X, \tilde{I})$ is defined to be the union of the sets of irreducible components of the members of the locally finite family that gives rise to it.

Definition 5. Let $C$ be an $r$-determined irreducible (locally) closed $(C)$-analytic subset of $J^{\infty}(X, \tilde{I})$. Its codimension $\operatorname{codim}\left(C, J^{\infty}(X, \tilde{I})\right)$ is defined to be the codimension of $\pi_{r}^{\infty}(C)$ in $J^{r}(X, \tilde{I})$.

The above definition does not depend on $r$ because for any two positive integers $s>r$, the mapping $\pi_{r}^{s}: J^{s}(X, \tilde{I}) \rightarrow J^{r}(X, \tilde{I})$ is an affine bundle and, therefore, preserves codimension and irreducibility by inverse image.

Consider a possibly infinite filtration

$$
C_{1} \supset \cdots \supset C_{i} \supset \ldots
$$

of closed $(C)$-analytic subsets of $J^{\infty}(X, \tilde{I})$. We say that an irreducible component 
$C_{i, j}$ of $C_{i}$ is stable if it is an irreducible component of $C_{k}$ for any $k \geq i$. The component $C_{i, j}$ is called strongly unstable if it does not contain any stable component of $C_{k}$ for any $k \geq i$. Let $\left\{C_{i, j}\right\}_{j \in \mathbb{N}}$ be the set of strongly unstable irreducible components of $C_{i}$ (if there is a finite amount we allow repetition in the indexing).

The intersection of all the closed subsets of the filtration decomposes naturally as

$$
\bigcap_{i \in \mathbb{N}} C_{i}=Z_{1} \cup Z_{2},
$$

where $Z_{1}$ is the union of all the stable irreducible components of the $C_{i}$ 's and

$$
Z_{2}:=\bigcup_{\left\{j_{i}\right\}_{i \in \mathbb{N}} \in \mathbb{N}^{\mathbb{N}}}\left(\bigcap_{i \in \mathbb{N}}^{\infty} C_{i, j_{i}}\right) .
$$

Take any positive integer $c$. Let $\left\{C_{j}^{\prime}\right\}_{j \in \mathcal{J}_{c}}$ be the set of all the strongly unstable components of any of the $C_{i}$ 's whose codimension is bigger or equal than $c$. Let $\mathcal{J}_{c}^{\prime}$ be the indexes corresponding to the components which are maximal by the inclusion relation among the elements of $\left\{C_{j}^{\prime}\right\}_{j \in \mathcal{J}_{c}}$. The following inclusion follows easily from the definition of strongly unstable components and from the fact that each $C_{i}$ is a locally finite union of irreducible closed $(C)$-analytic subsets:

$$
Z_{2} \subset \bigcup_{j \in \mathcal{J}_{c}^{\prime}} C_{j}^{\prime}
$$

It is easy to check that the family $\left\{C_{j}^{\prime}\right\}_{j \in \mathcal{J}_{c}^{\prime}}$ is locally finite. Hence the set $Z_{2}$ is contained in a closed $(C)$-analytic subset with all of its irreducible components of codimension bigger or equal than $c$. This motivates

Definition 6. A closed subset of $J^{\infty}(X, \tilde{I})$ is residual if for any positive integer $c$ it is contained in a closed $(C)$-analytic subset of $U$ with all its irreducible components of codimension at least $c$.

Remark 7. Suppose that $Z \subset J^{\infty}(X, \tilde{I})$ admits a decomposition as a union of a closed $(C)$-analytic subset $Z^{(a)}$ and a residual subset $Z^{(r)}$. The subset $Z^{(a)}$ is uniquely determined by $Z$, and is called the analytic part of $Z$. The subset $Z^{(r)}$ is uniquely determined if it is minimal among the subsets such that $Z=Z^{(a)} \cup Z^{(r)}$. Then it is called the residual part of $Z$.

Given a filtration like (9), the intersection of all its terms can be decomposed as the union of a closed $(C)$-analytic subset and a residual subset.

\section{The topological and finite-determinacy discriminants}

In order to fix ideas we state now what we mean by topological finite-determinacy of functions. 
Definition 8. Two germs of functions $f:\left(\mathbb{K}^{n}, x\right) \rightarrow(\mathbb{K}, t)$ and $f^{\prime}:\left(\mathbb{K}^{n}, x^{\prime}\right) \rightarrow$ $\left(\mathbb{K}, t^{\prime}\right)$ are said to be topologically right-left equivalent (we will say topologically equivalent to abbreviate) if there are germs of homeomorphisms $\phi:\left(\mathbb{K}^{n}, x^{\prime}\right) \rightarrow$ $\left(\mathbb{K}^{n}, x\right)$ and $\psi:(\mathbb{K}, t) \rightarrow\left(\mathbb{K}, t^{\prime}\right)$ such that $\psi \circ f \circ \phi=g$. A function $f \in \tilde{I}_{x}$ is called topologically $k$-determined with respect to $\tilde{I}$ if any other $g \in \tilde{I}_{x}$ with the same $k$-jet is topologically equivalent to $f$.

Proposition 9. Let $X$ be any $\tilde{I}$-stratum of $W$. Let $T \subset J^{\infty}(\tilde{I}, X)$ be a rdetermined locally closed irreducible $(C)$-analytic subset. There exists an $s \geq r$ and a proper s-determined closed $(C)$-analytic subset $A$ of $T$ such that if two germs $f, g \in T$ have their s-jets $\pi_{s}^{\infty}(f)$ and $\pi_{s}^{\infty}(g)$ in the same connected component of

$$
\pi_{s}^{\infty}(T) \backslash \pi_{s}^{\infty}(A)^{\prime}
$$

then they are topologically equivalent.

The proof of this proposition will be given in the last section of the paper.

Notation. Let $T$ be a subset of $J^{r}(X, \tilde{I})$ for a certain $r \leq \infty$. Given any $x \in W$, we denote by $T_{x}$ the fibre $\left(p r_{r}\right)^{-1}(x) \cap T$ of the restriction of the mapping $p r_{r}$ to $T$. If $V$ is a subset of $X$ we denote by $T_{\mid V}$ the intersection $\left(p r_{r}\right)^{-1}(V) \cap T$.

Let $T \subset J^{\infty}(\tilde{I}, X)$ be a locally closed $(C)$-analytic subset with irreducible components $\left\{T_{j}\right\}_{j \in J}$. For each $j \in J$, let $A_{j} \subset T_{j}$ be the $(C)$-analytic subset predicted by Proposition 9 . By the locally finiteness of the family $\left\{T_{j}\right\}_{j \in J}$, the union $A:=\cup_{j \in J} A_{j}$ is closed $(C)$-analytic in $T$. It is easy to check that any germ in $T \backslash A$ is topologically finite-determined and any two germs in the same path-connected component of it are topologically equivalent.

Proposition 10. Let $T$ be a locally closed $(C)$-analytic subset of $J^{\infty}(\tilde{I}, X)$. There exist unique subsets $\Gamma \subset \Delta$ of $T$ not containing any irreducible component of it with the following properties:

(i) We have decompositions $\Delta=\Delta^{(a)} \cup \Delta^{(r)}$ and $\Gamma=\Gamma^{(a)} \cup \Gamma^{(r)}$ where $\Delta^{(a)}$ and $\Gamma^{(a)}$ are closed $(C)$-analytic subsets, and $\Delta^{(r)}$ and $\Gamma^{(r)}$ are residual closed subsets.

(ii) Any $f, g \in T$ in the same path-connected component of $T \backslash \Gamma$ are topologically equivalent. Moreover, any $f \in T \backslash \Delta$ is topologically finite-determined with respect to $\tilde{I}$.

(iii) The subsets $\Delta$ and $\Gamma$ are minimal among the subsets of $T$ satisfying Properties (i) and (ii).

For any positive integer $k$, we let $T_{\leq k}$ be the union of the irreducible components of $T$ which are $k$-determined, and $T_{>k}$ be the union of all the other irreducible components. There is a unique subset $\Delta_{k}$ of $T_{\leq k}$ such that:

- It is the union of a closed $(C)$-analytic subset and a residual subset. 
- Any two germs in the same path-connected component of $T_{\geq k} \backslash \Delta_{k}$ are topologically $k$-determined and have the same topological type.

- It is minimal among the subsets of $T_{\leq k}$ having the three properties above.

Moreover, the subset $\Delta_{k}$ is actually a $k$-determined closed $(C)$-analytic.

We have $\Gamma \subset \Delta \subset \cap_{k \geq 0}\left(\Delta_{k} \cup T_{>k}\right)$. Moreover

$$
\Gamma^{(a)}=\Delta^{(a)}=\left(\cap_{k \geq 0}\left(\Delta_{k} \cup T_{>k}\right)\right)^{(a)} .
$$

In other words, the subsets $\Gamma, \Delta$ and the intersection $\cap_{k \geq 0}\left(\Delta_{k} \cup T_{>k}\right)$, only may differ in a residual set.

Proof. Let $\mathcal{C}$ be the set whose elements are subsets of $T$ not containing any of its irreducible components and satisfying the first two properties of $\Gamma$. The set $\mathcal{C}$ is not empty because the subset $A$, constructed in the last paragraph before the proposition, belongs to it. We consider in $\mathcal{C}$ the partial order given by inclusion. Consider a chain

$$
K_{1} \supset \cdots \supset K_{i} \supset \ldots
$$

of subsets of $\mathcal{S}$. We claim that the intersection $K:=\cap_{i \in \mathbb{N}} K_{i}$ belongs to $\mathcal{C}$.

By Property (i) each $K_{i}$ decomposes as $K_{i}:=K_{i}^{(a)} \cup K_{i}^{(r)}$, with $K_{i}^{(a)}$ a closed $(C)$-analytic subset and $K_{i}^{(r)}$ residual. The closed $(C)$-analytic parts form a chain

$$
K_{1}^{(a)} \supset \cdots \supset K_{i}^{(a)} \supset \ldots
$$

We construct a chain

$$
L_{1} \supset \cdots \supset L_{i} \supset \ldots
$$

of subsets that admit a decomposition in a closed $(C)$-analytic subset $L_{i}^{(a)}$ and a residual subset $L_{i}^{(r)}$ such that $\cap_{i \in \mathbb{N}} L_{i}=K$ and all the irreducible components of $L_{i}^{(a)}$ are either stable or of codimension at least $i$. We proceed inductively: suppose that for a certain positive integer $m$ we have defined a chain

$$
L_{1} \supset \cdots \supset L_{m} \supset L_{m, m+1} \supset \cdots \supset L_{m, m+i} \supset \ldots
$$

such that, for any $i \leq m$, all the irreducible components of $L_{i}^{(a)}$ are either stable or of codimension at least $i$ and

$$
\left(\bigcap_{i \leq m} L_{i}\right) \bigcap\left(\bigcap_{i>m} L_{m, i}\right)=K .
$$

Clearly, for any $j \in \mathbb{N}$ there are no non-stable components of codimension strictly smaller than $m$ in $L_{m, m+j}^{(a)}$. Let $\left\{C_{h}\right\}_{h \in H}$ be the collection of irreducible components of $L_{m, m+1}^{(a)}$ of codimension $m$ which are non-stable in the filtration given by the closed $(C)$-analytic parts of the elements of filtration (15). For any $h \in H$ we denote by $i_{h}$ the smallest positive integer such that $C_{h}$ is not an irreducible component of $L_{m, m+i_{h}}^{(a)}$. For any positive integer $j$ let $\left\{Z_{l}\right\}_{l \in L}$ be the set of irreducible 
components of $L_{m, m+j}$ not belonging to $\left\{C_{h}\right\}_{h \in H}$. Define

$$
L_{m+1, m+j}:=\left(\bigcup_{l \in L} Z_{l}\right) \bigcup L_{m, m+j}^{(r)} \bigcup\left(\bigcup_{j<i_{h}}\left(C_{h} \cap L_{m, m+i_{h}}\right)\right) .
$$

Define $L_{m+1}=L_{m+1, m+1}$. By construction, the Equality (16) holds replacing $m$ by $m+1$. We will prove that each subset $L_{m+1, m+j}$ for $j \geq 0$ admits a decomposition in a closed $(C)$-analytic subset $L_{i}^{(a)}$ and a residual subset $L_{i}^{(r)}$. By construction, all the non-stable irreducible components of $L_{m+1}^{(a)}$ are of codimension at least $m+1$. Iterating inductively the procedure we obtain the desired chain (14).

For any non-negative integer $j$ we consider the decomposition $L_{m+1, m+j}=$ $L_{m+1, m+j}^{(a)} \cup L_{m+1, m+j}^{(r)}$, where

- the set $L_{m+1, m+j}^{(a)}$ is the union of all the irreducible components of $L_{m, m+j}^{(a)}$ not belonging to $\left\{C_{h}\right\}_{h \in H}$, together with $\cup_{j<i_{h}}\left(C_{h} \cap L_{m, m+i_{h}}^{(a)}\right)$.

- the set $L_{m+1, m+j}^{(r)}$ is the union of the sets $L_{m, m+j}^{(r)}$ and $\cup_{j<i_{h}}\left(C_{h} \cap L_{m, m+i_{h}}^{(r)}\right)$.

As the irreducible components $\left\{C_{h}\right\}_{h \in H}$ form a locally finite family, the set $L_{m+1, m+j}^{(a)}$ is a locally finite union of closed $(C)$-analytic subsets and, hence, it is closed $(C)$-analytic. On the other hand, for any positive integer $c$ and any $h$ such that $j<i_{h}$, there exists a closed $(C)$-analytic subset $C_{h}^{\prime}$ contained in $C_{h}$ and containing $C_{h} \cap L_{m, m+i_{h}}^{(r)}$, with all its irreducible components of codimension at least $c$. By the local finiteness of $\left\{C_{h}\right\}_{h \in H}$, the subset $\cup_{j<i_{h}} C_{h}^{\prime}$ is closed $(C)$ analytic. Therefore, $\cup_{j<i_{h}}\left(C_{h} \cap L_{m, m+i_{h}}\right)^{(r)}$ is residual and, hence, $L_{m+1, m+j}^{(r)}$ is also residual.

Now we prove that $K$ belongs to $\mathcal{C}$. Let $K^{(a)}$ be the union of the stable irreducible components of the filtration given by the $L_{i}^{(a)}$ 's. Define $D$ to be the union of all the intersections of the form $\cap_{i \in \mathbb{N}}\left(L_{i}^{(u)} \cup L^{(r)}\right)$, where $L_{i}^{(u)}$ is the union of all the non-stable components of $L_{i}$. As $K=\cap_{i \in \mathbb{N}} L_{i}$, we obtain easily the decomposition $K=K^{(a)} \cup D$. We show that $D$ is residual: let $c$ be any positive integer. Consider a closed $(C)$-analytic subset $C$ containing $L_{c}^{(r)}$ with all its irreducible components of codimension at least $c$. The set $L_{c}^{(u)} \cup C$, whose irreducible components are all of them of codimension at least $c$, contains $D$.

Let $\gamma:[0,1] \rightarrow T \backslash K$ be a continuous path. In order to show that $K$ belongs to $\mathcal{C}$, we only have to check that the topological type of the germ $\gamma(t)$ is independent of $t$. The set $T \backslash K$ is the union of the increasing sequence of open subsets $\left\{T \backslash K_{i}\right\}_{i \in \mathbb{N}}$. By the compactness of $[0,1]$, there is an index so that $\gamma([0,1]) \subset T \backslash K_{i}$. As $K_{i} \in \mathcal{C}$, the topological type remains constant along $[0,1]$.

We have shown that any decreasing sequence in $\mathcal{C}$ has a lower bound. By Zorn's Lemma we deduce the existence of $\Gamma$. The uniqueness holds, as the intersection of two subsets in $\mathcal{C}$ is easily shown to belong to $\mathcal{C}$. The existence and uniqueness of $\Delta$ and $\Delta_{k}$ for any $k \in \mathbb{N}$ is analogous.

Now we show that $\Delta_{k}$ is $k$-determined $(C)$-analytic. Consider an irreducible component $Z$ of $\Delta_{k}^{(a)}$. Let $r$ be the determinacy degree of $Z$. If $r \leq k$ then 
$Z$ is $k$-determined. We study the case $r \geq k$. Consider the affine bundle $\pi_{k}^{r}$ : $J^{r}(X, \tilde{I}) \rightarrow J^{k}(X, \tilde{I})$. Let $B:=\pi_{k}^{\infty}\left(T_{\leq k}\right), E:=\pi_{r}^{\infty}\left(T_{\leq k}\right)$, and $\pi:=\pi_{k \mid E}^{r}: E \rightarrow B$. The irreducible closed $(C)$-analytic subset $Z_{r}:=\pi_{r}^{\infty}(Z)$ is contained in $E$. We claim that the set

$$
Y:=\left\{y \in B: E_{y} \subset Z_{r}\right\}
$$

is a (possibly empty) closed $(C)$-analytic subset of $B$. When $\mathbb{K}=\mathbb{R}$, it is easy to choose complexifications $E^{*}$ and $B^{*}$ of $E$ and $B$, and a mapping $\pi^{*}: E^{*} \rightarrow B^{*}$, which is a complex affine bundle, such that $\pi_{\mid E}^{*}=\pi$. As $Z_{r}$ is $C$-analytic, there exists an open neighbourhood $U^{*}$ of $E$ in $E^{*}$, and an irreducible complex closed analytic subset $Z_{r}^{*}$ of $U^{*}$ such that $Z_{r}^{*} \cap E=Z_{r}$. The open subset $U^{*}$ can be chosen so that for any $x \in B$ the fibre $U_{x}^{*}$ is connected (we prove this at the end). Let $N$ be the rank of the affine bundle $\pi^{*}: E^{*} \rightarrow B^{*}$. The subset

$$
A^{*}:=\left\{z \in Z^{*}: \operatorname{dim}_{z}\left(Z_{\pi^{*}(z)}^{*}\right)=N\right\}
$$

is a complex closed analytic subset of $Z^{*}$. Given any $z \in A^{*}$, we have $\operatorname{dim}_{z} Z_{\pi^{*}(z)}^{*}=$ $\operatorname{dim}_{z}\left(E_{\pi^{*}(z)}^{*}\right)$. Therefore, $Z_{\pi^{*}(z)}^{*}$ contains an open neighborhood $V_{z}$ of $z$ in $E_{\pi^{*}(z)}^{*}$. Clearly, $V_{z}$ is contained in $A_{\pi^{*}(z)}^{*}$ and, hence, this subset is both closed and open in the connected set $U^{*} \cap E_{\pi^{*}(z)}^{*}$. Thus $A_{\pi^{*}(z)}^{*}=U^{*} \cap E_{\pi^{*}(z)}^{*}$ for any $z \in A^{*}$. This implies

$$
U^{*} \cap\left(\pi^{*}\right)^{-1}\left(\pi^{*}\left(A^{*}\right)\right)=A^{*}
$$

and from here it is easy to deduce that $\pi^{*}\left(A^{*}\right)$ is a closed complex analytic subset of the open subset $\pi^{*}\left(U^{*}\right) \subset B^{*}$. For any $x \in B$ we have that $U^{*} \cap E_{x}^{*} \supset E_{x}$. Therefore $\pi^{*}\left(A^{*}\right) \cap B \subset Y$. On the other hand, if $x \in Y$, we have $E_{x} \subset U^{*} \cap Z_{x}^{*}$. Consequently, $Z_{x}^{*}$ is a closed complex analytic subset containing the real part $E_{x}$ of $U^{*} \cap E_{x}^{*}$. This implies that $Z_{x}^{*}$ contains a neighbourhood of $E_{x}$ in $U^{*} \cap E_{x}^{*}$ and, hence, by connectedness of $U^{*} \cap E_{x}^{*}$, it is equal to it. Thus, $\pi^{*}\left(A^{*}\right) \cap B=Y$, which proves our claim when $\mathbb{K}=\mathbb{R}$. The proof in the complex case is analogous, but easier.

If $\left(\pi_{k}^{\infty}\right)^{-1}(Y)=Z$, then $Z$ is actually $k$-determined. Otherwise we let $\Delta_{k}^{\prime}$ be the subset given by the union of $\Delta^{(r)},\left(\pi_{k}^{\infty}\right)^{-1}(Y)$, and all the irreducible components of $\Delta_{k}^{(a)}$ different from $Z$. Clearly, the set $\Delta_{k}^{\prime}$ is strictly contained in $\Delta_{k}$. Consider two germs $f, g$ in the same path-connected component of $T_{\leq k} \backslash \Delta_{k}^{\prime}$. We claim that both of them are topologically $k$-determined and have the same topological type. This clearly gives a contradiction with the minimality of $\Delta_{k}$, which shows that $Z$ is $k$-determined. In the special case that neither $f$ nor $g$ belong to $\Delta_{k}$, the claim holds by definition of $\Delta_{k}$. Suppose that $f$ belongs to $\Delta_{k}$. As $f$ does not belong to $\Delta_{k}^{\prime}$, there exists an open neighbourhood $V_{f}$ of $f$ in $J^{\infty}(X, \tilde{I})$ such that $V_{f} \cap \Delta_{k}^{\prime}=\emptyset$. Then $f$ must be an element of $Z \backslash\left(\pi_{k}^{\infty}\right)^{-1}(Y)$. Let $x:=p r_{\infty}(f)$. As $f$ does not belong to $\left(\pi_{k}^{\infty}\right)^{-1}(Y)$, there exists a continuous path $\gamma:[0,1] \rightarrow V_{f} \cap T_{\leq k} \cap J^{\infty}(X, \tilde{I})_{x}$ such that $\gamma(0)=f$, the $k$-jet of $\gamma(t)$ does not depend on $t$, and $\gamma(t)$ does not belong to $Z$ for $t \neq 0$. Obviously, $\gamma(t) \notin \Delta_{k}$ 
for $t \neq 0$, and therefore the claim is reduced to the already verified case in which neither $f$ nor $g$ belong to $\Delta_{k}$.

In order to show the $k$-determinacy of $\Delta_{k}$, it remains to show $\Delta_{k}^{(r)}=\emptyset$, but this can be proved using arguments analogous to the last paragraph.

The inclusions $\Gamma \subset \Delta \subset \cap_{k>0}\left(\Delta_{k} \cap T_{>k}\right)$ are trivial. Now we show Equality (11). Let $C$ be an irreducible component of $\left(\cap_{k}>0\left(\Delta_{k} \cup T_{>k}\right)\right)^{(a)}$. We suppose that $C$ is not contained in $\Gamma$, and we look for a contradiction. There exists a certain integer $r$ such that $C$ is an irreducible component of $\Delta_{k}$ for any $k \geq r$. This is so because, once we have chosen $r$ large enough, we can assume that $T_{>r}$ does not contain $C$. In particular $C$ is $r$-determined. By Proposition 9 we find an integer $s \geq r$ and a $s$-determined proper closed $(C)$-analytic subset $A_{1} \subset C$ such that any germ in $C \backslash A_{1}$ is $s$-determined. Define $\Delta_{s}^{\prime}$ as the union of all the irreducible components of $\Delta_{s}$ different from $C$. Let $A_{2}$ be the union of all the irreducible components of $\Gamma^{(a)}$ not contained in $\Delta_{s}^{\prime} \cup T_{>s}$. As $\Gamma$ is contained in $\Delta_{s} \cup T_{>s}$ we have the inclusion $A_{2} \subset C$; moreover the last inclusion is strict because $C$ is not contained in $\Gamma$. Consider a proper closed $(C)$-analytic subset $A_{3}$ of $C$ such that $\Gamma^{(r)}$ is contained in $A_{3} \cup \Delta_{s}^{\prime} \cup T_{>s}$ (the existence of $A_{3}$ is clear as $\Gamma^{(r)}$ is residual). Define

$$
A:=A_{1} \cup A_{2} \cup A_{3} \cup\left(C \cap T_{>s}\right) .
$$

Any germ in $T_{\leq s} \backslash\left(A \cup \Delta_{s}^{\prime}\right)$ belongs either to $T_{\leq s} \backslash \Delta_{s}$ or to $C \backslash A_{1}$. Therefore, it is topologically $s$-determined. The set $T_{\leq s} \backslash\left(A \cup \Delta_{s}^{\prime}\right)$ is clearly included in $T \backslash \Gamma$ and, hence, any two germs in the same path-connected component of $T_{\leq s} \backslash\left(A \cup \Delta_{s}^{\prime}\right)$ have the same topological type. Then $A \cup \Delta_{s}^{\prime}$ have the same properties of $\Delta_{s}$ and is strictly smaller than it. This is a contradiction.

Finally, let's check that $U^{*}$ can be chosen so that $U^{*} \cap E_{x}^{*}$ is connected for any $x \in B$. As $\pi: E \rightarrow B$ has contractible fibres there is a continuous section $s: B \rightarrow E$. Therefore, we can give a continuous $\mathbb{R}$-vector bundle structure to $\pi: E \rightarrow B$ and to $\pi^{*}: E^{*} \rightarrow B^{*}$ in such a way that $E$ is a subbundle of the restriction of $E^{*}$ to $B$. Shrinking enough $B^{*}$ around $B$ we can suppose that $B$ is a strong deformation retract of $B^{*}$. Indeed, by the Triangulation Theorem for real analytic subsets we can think of $\left(B^{*}, B\right)$ as a polyhedral pair; then we apply Corollary 11 of [16], page 124. By well known arguments it follows that there is a vector subbundle $q: F \rightarrow B^{*}$ of $\pi: E^{*} \rightarrow B^{*}$ extending $\pi: E \rightarrow B$. Using partitions of unity we can construct a continuous tensor on $E_{\mid B}^{*}$ that restricts to an euclidean inner product on each fibre $E_{x}$. Let $d_{x}$ be the distance induced by it in $E_{x}$. It is easy to find positive continuous functions

$$
\alpha: B^{*} \rightarrow \mathbb{R} \cup\{+\infty\} \quad \beta: B^{*} \rightarrow \mathbb{R},
$$

(where a basis of neighbourhoods for $+\infty$ in $\mathbb{R} \cup\{+\infty\}$ is given by $\{(a,+\infty)\}_{a \in \mathbb{R}}$ ), such that $\alpha(B)=\{+\infty\}$ and

$$
\left\{z \in E_{x}^{*}: d_{x}(z, 0)<\alpha(x), \quad d\left(z, F_{x}\right)<\beta(x)\right\} \subset U^{*} \cap E_{x}^{*}
$$


Redefining $U^{*}$ as the open subset

$$
\left\{z \in E^{*}: d_{\pi^{*}(z)}(z, 0)<\alpha\left(\pi^{*}(z)\right), \quad d\left(z, F_{\pi^{*}(z)}\right)<\beta\left(\pi^{*}(z)\right)\right\}
$$

we obtain the desired properties.

Definition 11. Let $T$ be a locally closed $(C)$-analytic subset of $J^{\infty}(\tilde{I}, X)$. We call the sets $\Gamma$ and $\Delta$ that were constructed in the last proposition topological and finite-determinacy discriminants of $T$ respectively. For any positive integer $k$ the set $\Delta_{k}$ is called the $k$-determinacy discriminant of $T$.

Next we study the behaviour of discriminants when restricting to open subsets of $U$ :

Lemma 12. Let $T, \Gamma$ and $\Delta$ be as in Proposition 10. Let $U$ be an open subset of $W$. When $\mathbb{K}=\mathbb{C}$, the subsets $\Gamma_{\mid U}$ and $T \cap \Delta_{\mid U}$ are respectively the topological and finite-determinacy discriminants of $T_{\mid U}$.

Proof. Clearly, $\Gamma_{\mid U}$ contains the topological discriminant of $T_{\mid U}$. Consider the decomposition $\Gamma_{\mid U}=\Gamma_{\mid U}^{(a)} \cup \Gamma_{\mid U}^{(r)}$, where the first component is $(C)$-analytic and the second is residual. Any irreducible component $C^{\prime}$ of $\Gamma_{\mid U}^{(a)}$ is a subset of a unique irreducible component $C$ of $\Gamma^{(a)}$, which is $r$-determined for a certain $r$. By Proposition 9 there exists $s \geq r$ and a $s$-determined closed $(C)$-analytic subset $A$ of $C$ such that any two germs in the same path-connected component of $C \backslash A$ have the same topological type. Let $Z$ be any irreducible component of $T$. As $\mathbb{K}=\mathbb{C}$ we have that both $Z \backslash \Gamma \cap Z$ and $C \backslash A$ are path-connected. Hence, two germs which are both contained in one of these two subsets have the same topological type. We claim that there exists a component $Z$ of $T$ containing $C$ such that the topological type of the germs of $Z \backslash \Gamma \cap Z$ is different to the topological type of the germs of $C \backslash A$. Otherwise we let $\Gamma_{1}^{\prime}$ be the union of all the irreducible components of $\Gamma^{(a)}$ different from $C$. Let $\Gamma_{2}^{\prime}$ be the union of the intersections $C \cap X$ where $X$ is any irreducible component of $T$ not containing $C$, and define

$$
\Gamma^{\prime}:=\Gamma_{1}^{\prime} \cup \Gamma_{2}^{\prime} \cup \Gamma^{(r)} \cup A .
$$

It is easy to check that $\Gamma^{\prime}$ has the first two properties of $\Gamma$ and is strictly smaller than it. This is a contradiction.

Let $Z^{\prime}$ be an irreducible component of $Z_{\mid U}$ containing $C^{\prime}$. As

$$
\operatorname{dim}\left(Z^{\prime}\right)=\operatorname{dim}(Z)>\operatorname{dim}(C)=\operatorname{dim}\left(C^{\prime}\right),
$$

the inclusion $C^{\prime} \subset Z^{\prime}$ is strict. As $Z^{\prime} \backslash \Gamma_{\mid U} \cap Z^{\prime}$ and $C^{\prime} \backslash\left(A \cap C^{\prime}\right)$ are contained in $Z \backslash \Gamma \cap Z$ and $C \backslash A$ respectively, the topological type of the germs of $Z^{\prime} \backslash \Gamma_{\mid U} \cap Z^{\prime}$ is different from the topological type of the germs of $C^{\prime} \backslash\left(A \cap C^{\prime}\right)$. It follows that $C^{\prime}$ is contained in the topological discriminant of $T_{\mid U}$.

The proof for $\Delta$ is analogous. 


\section{The main result}

For any $x \in W$ we denote by $\mathcal{D}_{x}$ the group of germs of analytic diffeomorphisms of $\mathbb{K}^{n}$ that fix $x$, and by $\mathcal{D}_{x, e}$ the set of germs of analytic diffeomorphisms at $x$ that not necessarily fix it. Following [9], we define $\mathcal{D}_{\tilde{I}_{x}, e}$ to be the subset of $\mathcal{D}_{x, e}$ preserving the ideal; i.e. the subset formed by the germs that have a representative $\phi: U \rightarrow W$ such that $\phi^{*} \tilde{I}_{\phi(y)}=\tilde{I}_{y}$ for any $y \in U$.

Let $\phi_{t}$ be a 1-parameter family of diffeomorphisms of $\mathcal{D}_{\tilde{I}_{x}, e}$ smoothly depending on $t$, such that $\phi_{0}=\operatorname{Id}_{\left(\mathbb{K}^{n}, x\right)}$. Let $\phi_{1, t}, \ldots, \phi_{n, t}$ be its components. The germ (at $x$ ) of analytic vector field defined by $X:=\sum_{i=1}^{t} d \phi_{i, t} / d t_{\mid t=0} \partial / \partial x_{i}$ preserves the ideal sheaf $\tilde{I}$; i.e., satisfies $X\left(\tilde{I}_{x}\right) \subset \tilde{I}_{x}$. Let $\Theta$ be the sheaf of analytic vector fields in $W$. Define $\Theta_{\tilde{I}, e}$ as the subsheaf whose sections preserve the ideal sheaf $\tilde{I}$. Denote the stalk of $\Theta_{\tilde{I}, e}$ at $x$ by $\Theta_{\tilde{I}_{x}, e}$. Integration associates to any $X \in \Theta_{x}$, a 1-parameter flow $\phi_{t}$ of germs of analytic diffeomorphisms of $\mathcal{D}_{x, e}$ for which $\phi_{0}=\operatorname{Id}_{\left(\mathbb{K}^{n}, x\right)}$; if $X \in \Theta_{\tilde{I}_{x}, e}$, then $\phi_{t} \in \mathcal{D}_{\tilde{I}_{x}, e}$ for any value of $t$.

Any representative $\phi: U \rightarrow W$ of a germ $\phi \in \mathcal{D}_{I_{x}, e}$ induces by pushforward a mapping

$$
\phi_{*}: J^{\infty}(U, \tilde{I}) \rightarrow J^{\infty}(\phi(U), \tilde{I}),
$$

defined by $\phi_{*}\left(f_{y}\right):=\left(f_{y} \circ \phi^{-1}\right)_{\phi(y)}$ for any $y \in U$ and $f_{y} \in \tilde{I}_{y}$. As the $\mathbf{m}_{y}$-adic filtration is transformed by pushforward into the $\mathbf{m}_{\phi(y)}$-adic filtration, the mapping $\phi_{*}$ descends to a mapping

$$
j^{r} \phi_{*}: J^{r}(U, \tilde{I}) \rightarrow J^{r}(\phi(U), \tilde{I}) .
$$

Clearly, any representative $\phi: U \rightarrow W$ of a germ $\phi \in \mathcal{D}_{I_{x}, e}$ preserves the HilbertSamuel stratification; i.e., $\phi(U \cap X)=\phi(U) \cap X$ for any $\tilde{I}$-stratum $X$. It is easy to check that the restriction

$$
j^{r} \phi_{*}: J^{r}(U \cap X, \tilde{I}) \rightarrow J^{r}(\phi(U) \cap X, \tilde{I})
$$

is an analytic diffeomorphism when $r<\infty$.

Definition 13. Let $X$ be a $\tilde{I}$-stratum of $W$, and $T \subset J^{\infty}(X, \tilde{I})$ be a (locally) closed $(C)$-analytic subset. We say that $T$ is flow-invariant if for any open subset $V \subset W$, any vector field $\theta \in \Gamma\left(V, \Theta_{\tilde{I}, e}\right)$ and any flow $\phi: U \times(a, b) \rightarrow V$ integrating $\theta$, we have $\phi_{t *}\left(T_{x}\right)=T_{\phi_{t}(x)}$ for any $t \in(a, b)$ and $x \in U$.

Now we are ready to state the main result of the paper:

Main Theorem. Shrink $W$ so that $\Theta_{\tilde{I}, e}$ is generated by sections defined on the whole $W$. Consider $X$, a $\tilde{I}$-statum of $W$. Let $T$ be any locally closed $(C)$-analytic subset of $J^{\infty}(X, \tilde{I})$. There exists

- a unique filtration (which we call the filtration by successive discriminants)

$$
T=A_{0} \supset A_{1} \supset \cdots \supset A_{i} \supset \ldots
$$


by closed $(C)$-analytic subsets,

- two residual subsets $\Gamma^{(r)}$ and $\Delta^{(r)}$ (called respectively the topological and finite-determinacy cumulative residual discriminants of $T$ ),

with the following properties:

(1) We have $\cap_{i \geq 0} A_{i} \subset \Gamma^{(r)} \subset \Delta^{(r)}$.

(2) For any $i \geq 0$, the sets $A_{i+1} \cup\left(\Gamma^{(r)} \cap A_{i}\right)$ and $A_{i+1} \cup\left(\Delta^{(r)} \cap A_{i}\right)$ are respectively the topological and finite-determinacy discriminants of $A_{i}$.

(3) Any irreducible component of $A_{i}$ has codimension at least $i$.

(4) If $T$ is flow-invariant, then $A_{i}$ is flow-invariant for any $i \geq 0$. Moreover the $k$-determinacy discriminant of $A_{i}$ is flow invariant. Therefore the sets $\Gamma^{(r)} \cap A_{i}$ and $\Gamma^{(r)} \cap A_{i}$ are contained in a residual subset which is an intersection of flow-invariant closed $(C)$-analytic subsets of $C$.

As a consequence, any germ $f$ of $T \backslash \Delta^{(r)}$ is topologically finite-determined with respect to $\tilde{I}$. Furthermore, if $\mathbb{K}=\mathbb{C}$, given any open subset of $W^{\prime} \subset W$, the filtration by successive discriminants and the topological and finite-determinacy cumulative residual discriminants for $T_{\mid W^{\prime}}$ are the restrictions over $W^{\prime}$ of the corresponding objects for $T$.

This theorem shows, in particular, that given any ideal sheaf of analytic functions, the subset of functions that are not topologically finite-determined with respect to it is very small (we can think of it as an infinite-codimension subset). In contrast with Theorem 1, we can not provide uniform finite-determinacy bounds for prescribed codimension, that is, we can not ensure that for a prescribed integer $i$, there is another positive integer $r$ for which the subset $A_{i}$ is necessarily $r$-determined. The reason is that in the jet-spaces $J^{r}(X, \tilde{I})$, instead of the algebraic structure present in ordinary jet-spaces, we have just an analytic structure. Furthermore, the subsets in which $\mathcal{O}_{\mathbb{C}^{n}, O}$ decomposes according to Varchenko's Theorem, are invariant by the whole $\mathcal{D}_{O}$. As we want to work in a neighbourhood of the origin, rather that just at the origin itself, we need to replace the $\mathcal{D}_{O}$-invariance by the flow-invariance. Nevertheless we have the following:

Remark 14. If we restrict ourselves to work at the origin, that is, to use the space $I=\tilde{I}_{O}$ instead of $J^{\infty}(X, \tilde{I})$, the corresponding jet spaces $I / \mathbf{m}_{O}^{r+1} \cap I$ are affine spaces. Then, if we assume $I$ to be generated by Nash functions, the arguments of this paper can be modified so that, if the starting subset $T$ of the Main Theorem is finitely determined and algebraic, then, the subsets $A_{i}$ are finitely-determined and algebraic. Finally, let $\mathcal{D}_{\tilde{I}, O}$ be the subgroup of $\mathcal{D}_{O}$ formed by diffeomorphisms which preserve the ideal $\tilde{I}_{O}$. If $T$ is assumed to be $\mathcal{D}_{\tilde{I}_{O}}$-invariant, then the subsets $\left\{A_{i}\right\}_{i \geq 0}, \Gamma^{(r)}$ and $\Delta^{(r)}$ can be constructed to be $\mathcal{D}_{\tilde{I}_{O}}$-invariant.

Proof of the Main Theorem. We show first the existence and uniqueness of the required objects satisfying all the requirements except Property 4. For any non- 
negative integer $j$ there is a unique filtration

$$
T=A_{0} \supset \cdots \supset A_{j}
$$

by closed $(C)$-analytic subsets, and two unique filtrations

$$
\begin{aligned}
& \Gamma_{0} \supset \cdots \supset \Gamma_{j} \\
& \Delta_{0} \supset \cdots \supset \Delta_{j}
\end{aligned}
$$

by closed subsets with the following properties:

- For any $i<j$ the sets $A_{i+1} \cup\left(\Gamma_{j} \cap A_{i}\right)$ and $A_{i+1} \cup\left(\Delta_{j} \cap A_{i}\right)$ are respectively the topological and finite-determinacy discriminants of $A_{i}$, being the set $A_{i}$ the analytic part in both cases.

- The sets $\Gamma_{j} \cap A_{j}$ and $\Delta_{j} \cap A_{j}$ are respectively the topological and finitedeterminacy discriminants of $A_{j}$.

The construction is obvious for $j=0$. Supposing that the filtrations have been constructed for a certain $j$, it is clear that $A_{j+1}$ must be defined to be equal to the non-residual part of the topological discriminant of $A_{j}$, that is

$$
A_{j+1}:=\left(\Gamma_{j} \cap A_{j}\right)^{(a)} .
$$

The set $\Gamma_{j+1}$ can only be defined to be the union of the residual parts of the topological discriminants of $A_{i}$, for any $i \leq j$, with the whole topological discriminant of $A_{j+1}$. The definition of $\Delta_{j+1}$ is analogous. We have shown by induction that the required filtrations can be constructed for any non-negative integer $j$ and are unique. It is easy to show that the infinite filtration

$$
A_{0} \supset \cdots \supset A_{i} \supset \ldots
$$

and the closed sets $\Gamma^{(r)}:=\cap_{j \in \mathbb{N}} \Gamma_{j}$ and $\Delta^{(r)}:=\bigcap_{j \in \mathbb{N}} \Delta_{j}$ satisfy Properties $1-3$ from the statement of the Theorem. If $\mathbb{K}=\mathbb{C}$, using Lemma 12 it is easy to check that the filtration by successive discriminants and the topological and finitedeterminacy cumulative residual discriminants satisfy the compatibility condition concerning restrictions to open subsets of $W$.

It only remains to prove Property 4 when $T$ is flow-invariant. We only prove the statement concerning $A_{i}$, being the one concerning the $k$-determinacy discriminant analogous. We work by induction on $i$. Suppose that $A_{j}$ is flow-invariant for any $j \leq i$. We show that $A_{i+1}$ is flow-invariant. For this we show that each irreducible component $C$ of $A_{i+1}$ is flow invariant.

Consider an increasing sequence $\left\{V_{k}\right\}_{k \in \mathbb{N}}$ of open subsets of $W$ such that the closure $\bar{V}_{k}$ is compact and contained in $V_{k+1}$ and the union $\cup_{k \in \mathbb{N}} V_{k}$ equals $W$. Denote by $\partial V_{k}$ the boundary $\bar{V}_{k} \backslash V_{k}$. Define $d_{k}:=d\left(\bar{V}_{k}, \partial V_{k+1}\right)$, that is, the minimal euclidean distance between points of $\bar{V}_{k}$ and $\partial V_{k+1}$. Consider a decreasing sequence $\left\{\epsilon_{k}\right\}_{k \in \mathbb{N}}$ of positive real numbers such that $\epsilon_{k}<d_{k}$ for any $k \in \mathbb{N}$.

Let $\mathcal{A}_{k}$ be the set of analytic diffeomorphisms $\phi$ such that there exists

- a vector field $\theta \in \Gamma\left(W, \Theta_{\tilde{I}, e}\right)$,

- an open subset $U$ which is a neighbourhood of $\bar{V}_{k}$, 
- a flow $\psi: U \times(a, b) \rightarrow W$ integrating $\theta$, such that $\phi=\psi_{t}$ for a certain $t \in(a, b)$.

For any $\phi \in \mathcal{A}_{k}$, we define $\delta(\phi):=\max \left\{\|\phi(x)-x\|: x \in \bar{V}_{k}\right\}$. Given any positive $\eta$, we define

$$
\mathcal{A}_{k}^{\eta}:=\left\{\phi \in \mathcal{A}_{k}: \delta(\phi)<\eta\right\}
$$

If $\eta \leq \epsilon_{k}$, then it is easy to show that $\phi\left(V_{k+1}\right) \supset \bar{V}_{k}$ for any $\phi \in \mathcal{A}_{k+1}^{\eta}$.

Let $r$ be the determinacy degree of the irreducible component $C$. The set

$$
D_{k}(\eta):=\bigcap_{\phi \in \mathcal{A}_{k+1}^{\eta}} \phi_{*}\left(C_{\mid V_{k+1}}\right)_{\mid V_{k}}
$$

is a $r$-determined closed $(C)$-analytic subset of $J^{\infty}\left(X \cap V_{k}, \tilde{I}\right)$ because it is intersection of such type of subsets. Let $\left\{C_{j}\right\}_{j \in J}$ be the irreducible components of $A_{i+1}$ different from $C$. Define

$$
A_{i+1, k}^{\prime}(\eta):=D_{k}(\eta) \bigcup\left(\bigcup_{j \in J} C_{j \mid V_{k}}\right) .
$$

Consider two germs $f$ and $g$ in the same path-connected component of

$$
A_{i \mid V_{k}} \backslash\left(A_{i+1, k}^{\prime}(\eta) \cup \Gamma_{\mid V_{k}}^{(r)}\right) .
$$

We claim that $f$ and $g$ are topologically equivalent. Define

$$
X:=\cap_{\phi \in \mathcal{A}_{k+1}^{\eta}} \phi_{*}\left(A_{i+1} \cup \Gamma^{(r)}\right)_{\mid V_{k}}
$$

As $X \subset A_{i+1, k}^{\prime}(\eta) \cup \Gamma_{\mid V_{k}}^{(r)}$, the germs $f$ and $g$ are in the same path-connected component of $A_{i \mid V_{k}} \backslash X$. Let $\gamma:[0,1] \rightarrow A_{i \mid V_{k}} \backslash X$ be a continuous path joining them. For each $t \in[0,1]$, there exists $\phi \in \mathcal{A}_{k+1}^{\eta}$ such that $\gamma(t)$ is not in the closed subset $\phi_{*}\left(A_{i+1} \cup \Gamma^{(r)}\right)_{\mid V_{k}}$. Therefore, there exists a positive $\xi$ such that $\gamma(t-\xi, t+\xi)$ does not meet $\phi_{*}\left(A_{i+1} \cup \Gamma^{(r)}\right)_{\mid V_{k}}$. Hence, $\phi^{*}(\gamma(t-\xi, t+\xi))$ is included in $A_{i} \backslash\left(A_{i+1} \cup \Gamma^{(r)}\right)$ and, consequently, all the germs of $\gamma(t-\xi, t+\xi)$ are topologically equivalent. Covering $[0,1]$ by intervals like $(t-\xi, t+\xi)$, we conclude the proof of our claim.

Now we show that $D_{k}(\eta)$ is flow invariant. We do it in two steps:

Step 1: we prove that $D_{k}(\eta)$ is flow-invariant with respect to vector fields defined on the whole $W$. Consider such a vector field $\theta \in \Gamma\left(W, \Theta_{I, e}\right)$. Choose a point $x \in V_{k}$; consider a neighbourhood $U$ of $x$ in $W$ and a flow $\psi: U \times(a, b) \rightarrow V_{k}$ (with $0 \in(a, b))$ obtained by integration of $\theta$. We have to show that $\psi_{t *} D_{k}(\eta)_{x}=$ $D_{k}(\eta)_{\psi_{t}(x)}$ for any $t \in(a, b)$.

In order to get lighter notation, denote by $D^{\prime}(\eta)$ the projection $\pi_{r}^{\infty}\left(D_{k}(\eta)\right)$. As $D_{k}(\eta)$ is $r$-determined, it is enough to show that $j^{r} \psi_{t *} D^{\prime}(\eta)_{x} \subset D^{\prime}(\eta)_{\psi_{t}(x)}$ for any $t \in(a, b)$ or, which is the same, that $j^{r} \psi_{t *}(f)$ belongs to $D^{\prime}(\eta)$ for any $f \in D^{\prime}(\eta)_{x}$. Choose $f \in D^{\prime}(\eta)_{x}$. Define $L_{f}$ to be the set whose elements are the numbers $t \in(a, b)$ such that $j^{r} \psi_{t *}(f)$ belongs to $D^{\prime}(\eta)$. As $D^{\prime}(\eta)$ is closed, the set 
$L_{f}$ is non-empty (as 0 belongs to it) closed subset of $(a, b)$. If we prove that $L_{f}$ is also open, then we conclude by connectedness of $(a, b)$.

Consider $t \in L_{f}$, we need to find a neighbourhood of $t$ included in $L_{f}$. As $\psi_{t_{1}} \circ \psi_{t_{2}}=\psi_{t_{1}+t_{2}}$, we can assume without loosing generality that $t=0$. By noetherianity of germs of analytic subsets, there exists a neighbourhood $N_{f}$ of $f$ in $J^{r}(X, \tilde{I})$ and a finite subset $\mathcal{A}(f)$ of $\mathcal{A}_{k+1}^{\eta}$ such that

$$
D^{\prime}(\eta) \cap N_{f}=\left[\bigcap_{\phi \in \mathcal{A}(f)} j^{r} \phi_{*}\left(\pi_{r}^{\infty}\left(C_{\mid V_{k+1}}\right)\right)_{\mid V_{k}}\right] \bigcap N_{f} ;
$$

By the finiteness of $\mathcal{A}(f)$, the number $\nu:=\max \{\phi \in \mathcal{A}(f): \delta(\phi)\}$ is strictly smaller than $\eta$. Consider the compact subset $K:=\cup_{\phi \in \mathcal{A}(f)} \phi\left(\bar{V}_{k+1}\right) \subset W$. There exists a positive $\xi$ such that if $|t|<\xi$, the domain of definition of $\psi_{t}$ contains an open neighbourhood of $K$ and

$$
\max \left\{\left\|\left(\psi_{t}\right)(x)-x\right\|: x \in K\right\}<\eta-\nu .
$$

Given any $\phi \in \mathcal{A}(f)$, and $t$ with $|t|<\xi$, the domain of definition of the composite $\psi_{t} \circ \phi$ is clearly a neighbourhood of $\bar{V}_{k+1}$. Moreover,

$$
\left\|\psi_{t} \circ \phi(x)-x\right\| \leq\left\|\psi_{t}(\phi(x))-\phi(x)\right\|+\|\phi(x)-x\|<\eta-\nu+\eta=\eta
$$

and, hence, $\psi_{t} \circ \phi$ belongs to $\mathcal{A}_{k+1}^{\eta}$. Then, we have

$$
\begin{aligned}
& j^{r} \psi_{t *}\left(D^{\prime}(\eta) \cap N_{f}\right)=j^{r} \psi_{t *}\left(\left[\bigcap_{\phi \in \mathcal{A}(f)} j^{r} \phi_{*}\left(\pi_{r}^{\infty}\left(C_{\mid V_{k+1}}\right)\right)_{\mid V_{k}}\right] \bigcap N_{f}\right) \subset \\
& \subset \bigcap_{\phi \in \mathcal{A}(f)} j^{r}\left(\psi_{t} \circ \phi\right)_{*}\left(\pi_{r}^{\infty}\left(C_{V_{\mid k+1}}\right)\right)_{\mid V_{k}} \subset \bigcap_{\phi \in \mathcal{A}_{k+1}^{\eta}} j^{r}(\phi)_{*}\left(\pi_{r}^{\infty}\left(C_{V_{k+1}}\right)\right)_{\mid V_{k}}=D^{\prime}(\eta)
\end{aligned}
$$

for any $t \in(-\xi, \xi)$; Therefore $(-\xi, \xi)$ is included in $L_{f}$.

Step 2: Let $\theta_{1}, \ldots, \theta_{l}$ be vector fields generating the sheaf $\Theta_{\tilde{I}, e}$ over $W$. Consider an open subset $U \subset W$ and a section $\theta \in \Gamma\left(U, \Theta_{\tilde{I}, e}\right)$. For any $x \in U$ there exists a neighbourhood $U_{x}$ of $x$ in $U$ and analytic functions $g_{1}, \ldots, g_{h}$ such that $\theta=\sum_{i=1}^{h} g_{i} \theta_{i}$. Choose local coordinates $\left(z_{1}, \ldots, z_{n}\right)$ around $x$ with the property that each $z_{i}$ is defined on the whole $\mathbb{K}^{n}$. Having perhaps to shrink $U_{x}$, we can assume that the power series expansions of $g_{1}, \ldots, g_{h}$ with respect to these coordinates are convergent on the whole $U_{x}$. Denote by $g_{i}^{(l)}$ the truncation of the power series expansion of $g_{i}$ at the $l$-th term. The functions $g_{i}^{(l)}$ are polynomials in the local coordinates and, hence, their domain of definition is also $\mathbb{K}^{n}$. Therefore, for each positive integer $l$ we can define the vector field $\theta^{(l)} \in \Gamma\left(W, \Theta_{\tilde{I}, e}\right)$ by the formula $\theta^{(l)}=\sum_{i=1}^{h} g_{i}^{(l)} \theta_{i}$. The sequence of vector fields $\left\{\theta^{(l)}\right\}_{l \in \mathbb{N}}$ converges to $\theta$ on $U_{x}$.

The following statement can be easily deduced from the continuous dependence of the solutions of differential equations with respect to a parameter (see [1] Ch. $1, \S 2.8$ ): there exists a positive integer $N$, an open neighbourhood $U_{x}^{\prime} \subset U_{x}$ of $x$ containing the origin, and a positive real number $\xi$ such that 
(1) for any $l \geq N$ there exists a flow $\psi^{(l)}: U_{x}^{\prime} \times(-\xi, \xi) \rightarrow U_{x}$ integrating the vector field $\theta^{(l)}$,

(2) there exists a flow $\psi: U_{x}^{\prime} \times(-\xi, \xi) \rightarrow U_{x}$ integrating the vector field $\theta$, and the sequence of mappings $\left\{\psi^{(l)}\right\}_{l \geq N}$ converges to $\psi$.

Suppose that we are given any flow $\psi: U^{\prime} \times(a, b) \rightarrow U$ (with $0 \in(a, b)$ ) integrating $\theta$. Define $L_{f}$ as in Step 1. Again, it is enough to show that $L_{f}$ is open. Using that $\psi_{t_{1}+t_{2}}=\psi_{t_{1}} \circ \psi_{t_{2}}$, the proof can be reduced to the existence of $\xi>0$ such that $(-\xi, \xi)$ is contained in $L_{f}$. Choose a positive $\xi$ and an open neighbourhood $U_{x}^{\prime} \subset U_{x}$ of $x$ such that the flow $\psi: U_{x}^{\prime} \times(-\xi, \xi) \rightarrow U$ is a limit of flows $\psi^{(l)}: U_{x}^{\prime} \times(-\xi, \xi) \rightarrow U$ integrating vector fields $\left\{\theta^{(l)}\right\}_{l \in \mathbb{N}}$ defined on the whole $W$. Then $j^{r} \psi_{t *}(f)$ is the limit of the sequence $\left\{j^{r} \psi_{t *}^{(l)}(f)\right\}_{l \in \mathbb{N}}$. For being $\theta^{(l)}$ defined on the whole $W$, due to Step 1 , we have $j^{r} \psi_{t *}^{(l)}(f) \in D^{\prime}(\eta)$ for any $l \in \mathbb{N}$ and any $t \in(-\xi, \xi)$. Then, as $D^{\prime}(\eta)$ is closed, we have $j^{r} \psi_{t *}(f) \in D^{\prime}(\eta)$ for any $t \in(-\xi, \xi)$. This concludes the proof of the flow-invariance of $D_{k}(\eta)$.

We claim that the restriction $D_{k+1}\left(\epsilon_{k+1}\right)_{\mid V_{k}}$ is equal to $D_{k}\left(\epsilon_{k}\right)$. As $V_{k+1} \subset V_{k+2}$ and $\epsilon_{k+1}<\epsilon_{k}$, we have $\mathcal{A}_{k+2}^{\epsilon_{k+1}} \subset \mathcal{A}_{k+1}^{\epsilon_{k}}$. Consequently, $D_{k+1}\left(\epsilon_{k+1}\right)_{\mid V_{k}} \supset D_{k}\left(\epsilon_{k}\right)$. Obviously

$$
\bigcap_{\phi \in \mathcal{A}_{k+1}^{\epsilon_{k}}} \phi_{*}\left(D_{k+1}\left(\epsilon_{k+1}\right)\right)_{\mid V_{k}} \subset \bigcap_{\phi \in \mathcal{A}_{k+1}^{\epsilon_{k}}} \phi_{*}\left(C_{\mid V_{k+1}}\right)_{\mid V_{k}}=D_{k}\left(\epsilon_{k}\right) .
$$

On the other hand, by the flow-invariance of $D_{k+1}\left(\epsilon_{k+1}\right)_{\mid V_{k}}$, the first term of the last expression is equal to $D_{k+1 \mid V_{k}}^{\epsilon_{k+1}}$. This shows our claim. It follows that the union

$$
D:=\bigcup_{k \in \mathbb{N}} D_{k}\left(\epsilon_{k}\right)
$$

is a $r$-determined closed $(C)$-analytic subset of $C$ which is flow-invariant. We define $A_{i+1}^{\prime}$ as the union of $D$ with all the irreducible components of $A_{i+1}$ different from $C$. Any two germs in the same connected component of $A_{i} \backslash\left(A_{i+1}^{\prime} \cup \Gamma^{(r)}\right)$ are topologically equivalent; indeed, it is enough to check this statement at the restriction over each $V_{k}$, and this has been already proved. Therefore, the set $A_{i+1}^{\prime} \cup\left(\Gamma^{(r)} \cap A_{i}\right)$ contains the topological discriminant of $A_{i}$. Taking analytic parts we get $A_{i+1}^{\prime} \supset A_{i+1}$, which implies that $D=C$. Consequently $C$ is flowinvariant.

\section{Generalization of Varchenko's method}

The overall structure of the proof of Proposition 9 follows [18]. It is based on an algorithm that shows the existence of a generic R-L-topological type for any family of functions, and on a definition of the so-called optimal germs in each finitedetermined locally closed analytic subset of $J^{\infty}(X, \tilde{I})$. Nevertheless, a straightforward generalization of Varchenko's proof to our case does not work, mostly 
because in his definition of optimal germs it is needed to perform certain modifications of functions that would take us outside the ideal sheaf we are working with. Also because he works with germs at the origin and we want to deal with a neighbourhood of it. This forces us to perform non-trivial modifications both in the algorithm and in the selection of optimal germs.

For notational convenience we recall $\S 1.1$ of $[18]$ : let $\mathbb{C}_{n}[z]$ be the space of monic polynomials $z^{n}+a_{n-1} z^{n-1}+\cdots+a_{0}$. It is an affine space whose coordinates are $a_{0}, \ldots, a_{n}$. For each sequence of positive integers $i_{1}, \ldots, i_{k}$ such that $i_{1}+\cdots+$ $i_{k}=n$, we consider the subset of $\mathbb{C}_{n}[x]$ consisting of polynomials with $k$ roots of multiplicities $i_{1}, \ldots, i_{k}$. This defines a stratification of $\mathbb{C}_{n}[z]$, whose strata will be called multiplicity strata. For each $m$, we define $S_{m}$ to be the union of multiplicity strata containing polynomials with less than $m$ different roots. The set $S_{m}$ is determined by a finite set of polynomial equations (with real coefficients) in $a_{0}, \ldots, a_{n}$.

Let $U \subset \mathbb{K}^{l}$ be an open subset. An $U$-family of functions is, by definition, a $\mathbb{K}$-valued analytic function $F$ defined on an open neighbourhood $V$ of $\{O\} \times U \subset$ $\mathbb{K}^{n} \times \mathbb{K}^{l}$. Let $T \subset U$ be a closed $(C)$-analytic analytic subset. A $T$-family of functions is the restriction to $V \cap\left(\mathbb{K}^{n} \times T\right)$ of a $U$-family of functions. With any $T$-family of functions, we associate its graph $\Gamma \subset\left[V \cap\left(\mathbb{K}^{n} \times T\right)\right] \times \mathbb{K}$, i.e. the subvariety defined by the function $P^{F}:=u-F$, where $u$ is the coordinate function of the target $\mathbb{K}$.

Suppose $\mathbb{K}=\mathbb{R}$. View $\mathbb{R}^{n}$ as the subset of points in $\mathbb{C}^{n}$ with real coordinates. Let $F$ be a $T$-family of functions. As $T$ is $C$-analytic, there exists an open subset $U^{*} \subset \mathbb{C}^{l}$ and a closed analytic subset $T^{*} \subset U^{*}$ such that $U^{*} \cap \mathbb{R}^{n}=U$ and $T^{*} \cap U=T$. According with Proposition 16 of [12], page 105, the subsets $T^{*}$ and $U^{*}$ can be chosen minimal in the following sense: if $T^{\prime}$ is any other complex analytic subset of a neighbourhood of $T$ in $\mathbb{C}^{n}$ for which $T^{\prime} \supset T$, then $T^{\prime} \cap W \supset T^{*} \cap W$ for a certain neighbourhood $W$ of $T$ in $\mathbb{C}^{n}$. As $F$ can be expressed locally as convergent power series, we can shrink $U^{*}$ so that there is a neighbourhood $V^{*}$ of $V$ in $\mathbb{C}^{n} \times \mathbb{C}^{l}$, containing $\{O\} \times U^{*}$, and a complex analytic function $F^{*}$ defined on $V^{*}$ whose restriction to $V$ is $F$. If $T^{*}$ is chosen minimal, we say that the $T^{*}$-family defined by $F^{*}$ is a minimal complex extension of the $T$-family defined by $F$. We denote by $\Gamma^{*}$ the graph of the $T^{*}$-family defined by $F^{*}$. Clearly $\Gamma^{*} \cap\left(\mathbb{R}^{n} \times \mathbb{R}^{l} \times \mathbb{R}\right)=\Gamma$.

Our aim is to show that generic functions of any $T$-family of functions are R-Ltopologically equivalent. We work first for $\mathbb{K}=\mathbb{C}$, and then explain the necessary modifications needed for the real case.

Algorithm. Fix a coordinate system $\left(x_{1}, \ldots, x_{n}\right)$ of $\mathbb{C}^{n}$. Consider a $T$-family of functions (for a certain $T \subset \mathbb{C}^{l}$ ). We describe an algorithm that constructs:

(i) a new coordinate system $\left(z_{1}, \ldots, z_{n}\right)$ of $\mathbb{C}^{n}$ (which will be called a suitable coordinate system),

(ii) a proper analytic subset $A \subset T$,

(iii) positive continuous functions $r_{1}, \ldots, r_{n+1}$ defined over $T \backslash A$,

(iv) non negative integers $k_{1}, k_{2}, \ldots, k_{n+1}$ (with $k_{1}=0$ ), 
(v) a sequence of pseudopolynomials $P_{1}, \ldots, P_{n+1}$ of the form $P_{n+1}:=u^{d_{n+1}}$, and

$$
P_{i}\left(z_{i}, \ldots, z_{n}, u, y\right)=z_{i}^{d_{i}}+\sum_{j=0}^{d_{i}-1} \alpha_{i}^{j}\left(z_{i+1}, \ldots, z_{n}, u, y\right) z_{i}^{j}
$$

for $i \leq n$, with $d_{i} \geq 0$ for any $i$, and $\alpha_{i}^{j}$ analytic in $U_{i+1}$, where

$$
U_{i}:=\left\{\left(z_{i}, \ldots, z_{n}, u, y\right): y \in T \backslash A,\left|z_{i}\right|<r_{i}(y), \ldots,\left|z_{n}\right|<r_{n}(y),|u|<r_{n+1}(y)\right\},
$$

with the following properties: let $\Gamma_{i}:=V\left(P_{i}\right) \cup V\left(R_{i}\right)$, where $R_{i}:=u^{k_{i}}$, then

(1) $\Gamma_{1} \cap U_{1}=\Gamma \cap U_{1}$.

(2) For each $i \leq n$ the polynomials $P_{i}\left(z_{i}, a_{i+1}, \ldots, a_{n}, b, c\right)$ are in the same multiplicity stratum as polynomials in $z_{i}$ if $\left(a_{i+1}, \ldots, a_{n}, b, c\right) \in U_{i+1} \backslash \Gamma_{i+1}$.

(3) The roots of the polynomial $P_{i}\left(z_{i}, a_{i+1}, \ldots, a_{n}, b, c\right)$ are in the disc of radius $r_{i}(c)$ for any $\left(a_{i+1}, \ldots, a_{n}, b, c\right) \in U_{i+1}$.

(4) $\alpha_{i}^{j}(0, \ldots, 0, y)=0$ for any $i, j$.

Notation 15. Let $f \in \mathbb{C}\left\{z_{1}, \ldots, z_{n}, u\right\}$, we define

$\operatorname{mult}^{\prime}(f):=\operatorname{mult}\left(f\left(z_{1}, \ldots, z_{n}, 0\right)\right), \quad$ and $\operatorname{wideg}_{z_{1}}(f):=\operatorname{mult}\left(f\left(z_{1}, 0, \ldots, 0\right)\right)$.

Now we describe the algorithm under the assumption that $P^{F}(0, \ldots, 0, y)=0$ for any $y \in T$ (otherwise the existence of the claimed objects is easy):

Step 1: As $P^{F}(0, \ldots, 0, y)=0$ for any $y \in T$, and $u \backslash P^{F}$, we deduce that $0<\operatorname{mult}^{\prime}\left(P^{F}(\cdot, \ldots, \cdot, y)\right)<\infty$ for any $y \in T$. Define

$$
d_{1}:=\min \left\{\operatorname{mult}^{\prime}\left(P^{F}(\cdot, \ldots, \cdot, y)\right): y \in T\right\} .
$$

Considering new coordinates $\left(z_{1}, z_{2}^{1} \ldots, z_{n}^{1}\right)$ of $\mathbb{C}^{n}$ related to $\left(x_{1}, \ldots, x_{n}\right)$ by the formulas $x_{1}:=z_{1}, x_{i}:=z_{1}^{i}+\lambda_{i}^{1} z_{1}$ for $i>1$, where the $\lambda_{i}^{1}$ 's are generic, we deduce that $\operatorname{wideg}_{z_{1}}\left(P^{F}(., \ldots, ., y)\right)=d_{1}$ for certain $y \in T$.

Define

$$
A_{1}:=\left\{y \in T: \operatorname{wideg}_{z_{1}}\left(P^{F}(\cdot, \ldots, \cdot, y)>d_{1}\right\} .\right.
$$

Clearly $A_{1}$ is a proper analytic subset of $T$.

By Weierstrass Preparation Theorem applied in a neighbourhood of the set

$$
\left\{(0, \ldots, 0, y): y \in T \backslash A_{1}\right\}
$$

there exists a neighbourhood $V_{1}$ of $T \backslash A_{1}$ in $\mathbb{C}^{l}$, and positive continous functions $r_{1}, r_{2}^{1}, \ldots, r_{n+1}^{1}$ defined on $V_{1}$ such that $P^{F}$ decomposes on the set

$$
\left\{\left(z_{1}, z_{2}^{1}, \ldots, z_{n}^{1}, u, y\right): y \in V_{1},\left|z_{1}\right|<r_{1}(y), \ldots,\left|z_{n}^{1}\right|<r_{n}^{1}(y),|u|<r_{n+1}^{1}(y)\right\}
$$

as $P^{F}=\phi P_{1}$, where

- the function $\phi$ is analytic and does not vanish at any point of the set and 
- $P_{1}$ is a pseudopolynomial of the form

$$
P_{1}\left(z_{1}, z_{2}^{1} \ldots, z_{n}^{1}, u, y\right)=z_{1}^{d_{1}}+\sum_{j=0}^{d_{1}-1} \alpha_{1}^{j}\left(z_{2}^{1}, \ldots, z_{n}^{1}, u, y\right) z_{1}^{j},
$$

such that its coefficients $\alpha_{1}^{j}$ are analytic on the set

$$
\left\{\left(z_{2}^{1}, \ldots, z_{n}^{1}, u, y\right): y \in V_{1},\left|z_{2}^{1}\right|<r_{2}^{1}(y), \ldots,\left|z_{n}^{1}\right|<r_{n}^{1}(y),|u|<r_{n+1}^{1}(y)\right\} .
$$

Set $k_{1}=0$. Choosing $r_{2}^{1}, \ldots, r_{n+1}^{1}$ small enough we ensure that Property 3 is satisfied for $P_{1}$.

Step $\mathbf{i}$ (for $1<i \leq n$ ): in the previous step we have constructed a system of coordinates $z_{1}, \ldots z_{i-1}, z_{i}^{i-1}, \ldots, z_{n}^{i-1}$ of $\mathbb{C}^{n}$, a proper analytic subset $A_{i-1} \subset$ $T$, positive continuous functions $r_{1}, \ldots r_{i-1}, r_{i}^{i-1}, r_{n+1}^{i-1}$, a neighbourhood $V_{i-1}$ of $T \backslash A_{i-1}$ in $\mathbb{C}^{l}$ and a pseudopolynomial $P_{i-1}=z_{i-1}^{d_{i-1}}+\sum_{j=0}^{d_{i-1}-1} \alpha_{i-1}^{j} z_{i-1}^{j}$ such that the functions $\alpha_{i-1}^{j}$ are analytic on the set $U_{i}^{\prime \prime}$ defined by

$$
\begin{aligned}
\left\{\left(z_{i}^{i-1}, \ldots, z_{n}^{i-1}, u, y\right): y \in V_{i-1},\left|z_{i}^{i-1}\right|\right. & <r_{i}^{i-1}(y), \ldots,\left|z_{n}^{i-1}\right| \\
& \left.<r_{n}^{i-1}(y),|u|<r_{n+1}^{i-1}(y)\right\} .
\end{aligned}
$$

Consider $P_{i-1}$ as a family of polynomials of $\mathbb{C}_{d_{i-1}}\left[z_{i-1}\right]$ parametrized by the set $U_{i}^{\prime}$ defined by

$$
\left\{\left(z_{i}^{i-1}, \ldots, y\right): y \in T \backslash A_{i-1},\left|z_{i}^{i-1}\right|<r_{i}^{i-1}(y), \ldots,\left|z_{n}^{i-1}\right|<r_{n}^{i-1}(y),|u|<r_{n+1}^{i-1}(y)\right\} .
$$

Since $T$ is irreducible there exists a multiplicity stratum of $\mathbb{C}_{d_{i-1}}\left[z_{i-1}\right]$ whose closure contains all the polynomials of this family, and such that there is a polynomial of the family belonging precisely to this stratum. Let this stratum contain polynomials with $m_{i}$ different roots. Let $G_{1}, \ldots, G_{k_{i}}$ be the polynomials in the variables $a_{0}, \ldots a_{d_{i-1}}$ determining the set $S_{m_{i}}$ in $\mathbb{C}_{d_{i-1}}\left[z_{i-1}\right]$. We order them so that the first $s_{i}$ of them (for a certain positive $s_{i}$ ) are the polynomials that does not vanish identically in $U_{i}^{\prime}$ when we substitute the variables $a_{j}$ 's by the functions $\alpha_{i-1}^{j}$ 's. Define an analytic function on the set $U_{i}^{\prime \prime}$ by the formula

$$
P_{i}^{\prime}:=\prod_{j=1}^{s_{i}} G_{j}\left(\alpha_{i-1}^{0}, \ldots, \alpha_{i-1}^{d_{i-1}-1}\right) .
$$

Having perhaps to substitute $r_{n+1}^{i-1}$ by another smaller positive continuous function, we can assume that $P_{i}^{\prime}$ admits a unique expression in $U_{i}^{\prime \prime}$ as

$$
P_{i}^{\prime}=\sum_{k=0}^{\infty} \psi_{k} u^{k}
$$

where $\psi_{k}$ is an analytic function on $U_{i}^{\prime \prime}$ not depending on $u$. Let $k_{i}^{\prime}$ be minimal such that the restriction of $\psi_{k_{i}^{\prime} \mid U_{i}^{\prime}}$ is not identically zero. Define $P_{i}^{\prime \prime}:=\sum_{k=k_{i}^{\prime}}^{\infty} \psi_{k} u^{k}$. Clearly $P_{i \mid U_{i}^{\prime}}^{\prime \prime}=P_{i \mid U_{i}^{\prime}}^{\prime}$. Define $k_{i}:=k_{i-1}+k_{i}^{\prime}$, and $P_{i}^{\prime \prime \prime}:=P_{i}^{\prime \prime} / u^{k_{i}^{\prime}}$. Clearly we have

$$
d_{i}:=\min \left\{\operatorname{mult}^{\prime}\left(P_{i}^{\prime \prime \prime}(0, \ldots, 0, y)\right): y \in T \backslash A_{i-1}\right\}<\infty .
$$


We consider several cases:

CASE $1\left(d_{i}=0\right)$ : We choose the definitive coordinate system $\left(z_{1}, \ldots, z_{n}\right)$ equal to $\left(z_{1}, \ldots, z_{i-1}, z_{i}^{i-1}, \ldots, z_{n}^{i-1}\right)$. Define the closed analytic proper subset $A$ as

$$
A:=A_{i-1} \cup\left\{y \in T \backslash A_{i-1}: \operatorname{mult}^{\prime}\left(P_{i}^{\prime \prime \prime}(\cdot, \ldots, \cdot, y)>0\right\} .\right.
$$

Define $P_{i}=\cdots=P_{n+1}=1$, take $k_{n+1}=\cdots=k_{i+1}=0$ and choose, for any $j \geq i$, the positive continuous function $r_{j}$ upper-bounded by $r_{j}^{i-1}$ and small enough so that the intersection $\left\{P_{i}^{\prime \prime}=0\right\} \cap U_{i}$ is empty. The algorithm concludes here.

CASE $2\left(d_{i}>0\right)$ : We consider new coordinates $\left(z_{1}, \ldots, z_{i}, z_{i+1}^{i}, \ldots, z_{n}^{i}\right)$ related to the previous ones by the formulas $z_{i}^{i-1}=z_{i}$ and $z_{j}^{i-1}=z_{j}^{i}+\lambda_{j}^{i} z_{i}$ for $j>i$. Choosing the $\lambda_{j}^{i}$ 's generic, we obtain that the set

$$
A_{i}:=A_{i-1} \cup\left\{y \in T \backslash A_{i-1}: \operatorname{wideg}_{z_{i}}\left(P_{i}^{\prime \prime \prime}\right)>d_{i}\right\}
$$

is a proper analytic subset of $T$.

By Weierstrass Preparation Theorem applied in a neighbourhood of the set

$$
\left\{(0, \ldots, 0, y): y \in T \backslash A_{i}\right\}
$$

there exists a neighbourhood $V_{i}$ of $T \backslash A_{i}$ in $\mathbb{C}^{l}$ and positive continuous functions $r_{i}, r_{i+1}^{i} \ldots, r_{n+1}^{i}$ such that $P_{i}^{\prime \prime}$ decomposes on the set

$$
\left\{\left(z_{i}, z_{i+1}^{i}, \ldots, z_{n}^{i}, u, y\right): y \in V_{i},\left|z_{i}\right|<r_{i}(y), \ldots,\left|z_{n}^{i}\right|<r_{n}^{i}(y),|u|<r_{n+1}^{i}(y)\right\}
$$

as $P_{i}^{\prime \prime}=\phi P_{i}$, where

- the function $\phi$ is analytic and does not vanish at any point of the set,

- $P_{i}$ is a pseudopolynomial of the form

$$
P_{i}\left(z_{i}, z_{i+1}^{i} \ldots, z_{n}^{i}, u, y\right)=z_{i}^{d_{i}}+\sum_{j=0}^{d_{i}-1} \alpha_{i}^{j}\left(z_{i+1}^{i}, \ldots, z_{n}^{i}, u, y\right) z_{i}^{j}
$$

such that its coefficients $\alpha_{i}^{j}$ are analytic on

$$
\left\{\left(z_{i+1}^{i}, \ldots, z_{n}^{i}, u, y\right): y \in V_{i},\left|z_{i+1}^{i}\right|<r_{i+1}^{i}(y), \ldots,\left|z_{n}^{i}\right|<r_{n}^{i}(y),|u|<r_{n+1}^{i}(y)\right\} .
$$

Choosing $r_{i+1}^{i}, \ldots, r_{n+1}^{i}$ small enough we ensure that Property 3 is satisfied for $P_{i}$.

Step $\boldsymbol{n}+1$ : this step runs parallel to the induction step (Step $i)$. As $u$ is the only variable of $P_{i+1}^{\prime}$ we must be in Case 1 . This concludes the algorithm.

The real case: Suppose now that $\mathbb{K}=\mathbb{R}$. Consider $T \subset U$, a closed $C$-analytic subset contained in an open subset of $\mathbb{R}^{n}$. Let $V$ be an open neighbourhood of $\{O\} \times U$ in $\mathbb{R}^{n} \times \mathbb{R}^{l}$. Let $F: V \rightarrow \mathbb{R}$ define a $T$-family of functions. Consider $T^{*} \subset U^{*} \subset \mathbb{C}^{n}$ and a complex analytic function $F^{*}: V^{*} \rightarrow \mathbb{C}$ (where $V^{*}$ is a neighbourhood of $\{O\} \times U^{*}$ in $\left.\mathbb{C}^{n} \times \mathbb{C}^{l}\right)$ such that the $T^{*}$-family given by $F$ is a minimal complex extension of the $T$-family defined by $F$. It is easy to check that the preceding algorithm can be applied to the $T^{*}$-family $F^{*}$ so that: 
(1) The initial coordinate system $\left(x_{1}, \ldots, x_{n}\right)$ is a real coordinate system when restricted to $\mathbb{R}^{n}$. In each step we choose a change of coordinates with real matrix, so that the new coordinate functions $\left(z_{1}, \ldots, z_{n}\right)$ of $\mathbb{C}^{n}$ form a real coordinate system when restricted to $\mathbb{R}^{n}$.

(2) The subset $A \cap \mathbb{R}^{n}$ is a proper $C$-analytic subset of $T$.

(3) For any $i \leq n$ and $j \leq d_{i}-1$, the function $\alpha_{i}^{j}$ assumes real values when $z_{i}, \ldots, z_{n}, u, y$ are real.

Given a $T$-family of functions $F$, for any $t \in T$ we will denote by $F_{\mid t}$ the germ at the origin given by the restriction of $F$ to $\mathbb{K}^{n} \times\{t\}$.

Proposition 16. Let $\mathbb{K}$ be either $\mathbb{R}$ or $\mathbb{C}$. Let $F$ be a $T$-family of functions. Let $A \subset T$ be the subset constructed in the preceding algorithm. For any $t, t^{\prime}$ in the same connected component of $T \backslash A$, the functions $F_{\mid t}$ and $F_{\mid t^{\prime}}$ are topologically $R$-L-equivalent.

Proof. Suppose $\mathbb{K}=\mathbb{C}$. Let $\Gamma$ be the graph of $F$. View $\Gamma$ as a family of analytic hypersurfaces parametrized by $T$. Proposition 3.1 of [18] can be adapted to apply (with minor changes in its proof) to this setting. If $\mathbb{K}=\mathbb{R}$, we consider a $T^{*}$ family $F^{*}$ which is a minimal complex extension of the $T$-family $F$. Let $\Gamma^{*}$ be the graph of $F^{*}$. Then, Proposition 3.2 of [18] can be adapted to apply (with changes in its proof) to the family of hypersurfaces $\Gamma^{*}$.

Notice that we have designed our algorithm so that the matrix $\left(c_{i, j}\right)$ relating the coordinate systems $\left(x_{1}, \ldots, x_{n}, u\right)$ and $\left(z_{1}, \ldots, z_{n}, u\right)$ of $\mathbb{K}^{n} \times \mathbb{K}$ has block form $c_{n+1, i}=c_{i, n+1}$ for any $i \neq n+1$. Since we are able to adapt Propositions 3.1 and 3.2 of [18], the proofs of Propositions 4.1 and 4.2 of [18] apply word by word in our case. Applying them respectively in the complex and real case, our result follows.

Let $X$ be any $\tilde{I}$-stratum of $W$. Let $T \subset J^{\infty}(X, \tilde{I})$ be an irreducible $(C)$ analytic subset. Before proving Proposition 9 , we have to distinguish a special class of germs in $T$, which will be called optimal germs. This class is, in a certain sense, "finitely determined and open". We select the germs that we will call optimal in the following way:

Search for Optimal Germs. The differences between the algorithm described above and Varchenko's algorithm force us to also introduce some different features in the selection of optimal germs. In particular, Varchenko's search for optimal germs can be performed with any coordinate system of $\mathbb{K}^{n}$. This will not be the case in our situation: as the search for optimal germs advances, we will need to modify our original coordinate system, getting at the end a new one that will be regarded as good coordinate system with respect to $T$. We will proceed in several stages in which we will select smaller subsets of $T$ each time.

We fix an initial coordinate system $\left(x_{1}, \ldots, x_{n}\right)$ for $\mathbb{K}^{n}$. We view any $f \in$ 
$J^{\infty}(X, \tilde{I})$ as a convergent power series in $\mathbb{C}\left\{x_{1}, \ldots, x_{n}\right\}$ by taking its Taylor expansion at $p r_{\infty}(f)$.

Stage 1. Given any $f \in T$ we consider $P^{f}=u-f \in \mathbb{C}\left\{x_{1}, \ldots, x_{n}, u\right\}$. Obviously, mult ${ }^{\prime}\left(P^{f}\right)<\infty$ and, therefore,

$$
d_{1}:=\min \left\{\operatorname{mult}^{\prime}\left(P^{f}\right): f \in T\right\}<\infty .
$$

Considering $z_{1}, z_{2}^{1}, \ldots, z_{n}^{1}$, a new coordinate system of $\mathbb{K}^{n}$ related with the old one by formulas of the form $x_{1}=z_{1}, x_{i}=z_{i}^{1}+\lambda_{i}^{1} z_{1}$, with the $\lambda_{i}^{1}$ 's real and generic enough, we obtain that $\min \left\{\operatorname{wideg}_{z_{1}}\left(P^{f}\right): f \in T\right\}=d_{1}$. Define the non-empty set

$$
\mathcal{E}_{1}^{1}:=\left\{f \in T / \operatorname{wideg}_{z_{1}}\left(P^{f}\right)=d_{1}\right\} .
$$

By Weierstrass Preparation Theorem, given any $f \in \mathcal{E}_{1}^{1}$, it is possible to find positive numbers $r_{1}(f), \ldots, r_{n+1}(f)$ such that $P^{f}$ can be decomposed over the open subset

$$
U_{1}^{1}(f):=\left\{\left(z_{1}, z_{2}^{1}, \ldots, z_{n}^{1}, u\right):\left|z_{1}\right| \leq r_{1}(f), \ldots,|u| \leq r_{n+1}(f)\right\}
$$

as $P^{f}=\phi_{1} P_{1}[f]$, where $\phi_{1}$ does not vanish anywhere in $U_{1}$ and $P_{1}[f]$ is a pseudopolynomial of the form

$$
P_{1}[f]\left(z_{1}, \ldots, u\right):=z_{1}^{d_{1}}+\sum_{j=1}^{d_{1}-1} z_{1}^{j} \alpha_{1}^{j}[f]\left(z_{2}^{1}, \ldots, u\right) .
$$

View $P_{1}[f]$ as a family of polynomials of $\mathbb{C}_{d_{1}}\left[z_{1}\right]$ (even when $\mathbb{K}=\mathbb{R}$ ) parametrized by the open subset

$$
U_{2}^{1}(f):=\left\{\left(z_{2}^{1}, \ldots, z_{n}^{1}, u\right):\left|z_{2}^{1}\right| \leq r_{2}(f), \ldots,|u| \leq r_{n+1}(f)\right\} .
$$

There exists a stratum $S_{1}(f)$ of $\mathbb{C}_{d_{1}}\left[z_{1}\right]$ whose closure contains the whole family, and such that there is a member of the family belonging to it. Let $m_{1}(f)$ be the number of roots of a generic element of $S_{1}(f)$. Define $m_{1}^{0}:=\max \left\{m_{1}(f): f \in \mathcal{E}_{1}^{1}\right\}$, and let $S_{1}^{0}$ be a stratum of $\mathbb{C}_{d_{1}}\left[z_{1}\right]$ such that there is $f \in \mathcal{E}_{1}^{1}$ with $S_{1}(f)=S_{1}^{0}$ and $m_{1}(f)=m_{1}^{0}$. Define the non-empty set

$$
\mathcal{E}_{1}^{2}:=\left\{f \in \mathcal{E}_{1}^{1} / S_{1}(f):=S_{1}^{0}\right\} .
$$

Let $z_{1}^{d_{1}}+\sum_{j=1}^{d_{1}-1} a_{j} z_{1}^{j}$ be a generic polynomial $\mathbb{C}_{d_{1}}\left[z_{1}\right]$. There are polynomials $Q_{1}, \ldots, Q_{s}$ (with real coefficients) in the variables $a_{0}, \ldots, a_{d_{1}-1}$ whose set of common zeros determines the set of polynomials in $\mathbb{C}_{d_{1}}\left[z_{1}\right]$ with less than $m_{1}^{0}$ roots. Let $f \in \mathcal{E}_{1}^{2}$. Denote by $T_{i}[f]$ the analytic functions in $z_{2}^{1}, \ldots, z_{n}^{1}, u$ obtained substituting in $Q_{1}$ the $a_{j}$ 's by the $\alpha_{1}^{j}[f]$ 's. Define $s_{1}(f)$ to be the number of $T_{j}[f]$ 's that do not vanish identically in $U_{2}(f)$. Clearly, $s_{1}(f)>0$ for any $f \in \mathcal{E}_{1}^{2}$. Define $s_{1}^{0}:=\max \left\{s_{1}(f): f \in \mathcal{E}_{1}^{2}\right\}$. Choose $f \in \mathcal{E}_{1}^{2}$ such that $s_{1}(f)=s_{1}^{0}$. Up to a re-ordering we can assume that

$$
P_{2}^{\prime}[f]:=\prod_{i=1}^{s_{1}^{0}} T_{i}[f]
$$


does not vanish identically in $U_{2}(f)$. Define the non-empty set

$$
\mathcal{E}_{1}^{3}:=\left\{f \in \mathcal{E}_{2}^{1} / P_{2}^{\prime}[f]_{\mid U_{2}(f)} \not \equiv 0\right\} .
$$

For any $f \in \mathcal{E}_{1}^{3}$ we let $k_{1}(f)$ be the maximal power of $u$ which divides $P_{2}^{\prime}[f]$. We define $k_{1}^{0}:=\min \left\{k_{1}(f): f \in \mathcal{E}_{1}^{3}\right\}$,

$$
\mathcal{E}_{1}:=\left\{f \in \mathcal{E}_{3}^{1} / k_{1}(f)=k_{1}^{0}\right\}
$$

and, for any $f \in \mathcal{E}_{1}$,

$$
P_{2}^{\prime \prime}[f]:=P_{2}^{\prime}[f] / u^{k_{1}^{0}} .
$$

Stage $\mathbf{i}$ (for $1<i \leq n$ ). In the previous stage we have chosen a coordinate system $z_{1}, \ldots, z_{i-1}, z_{i}^{i-1}, \ldots, z_{n}^{i-1}$ of $\mathbb{K}^{n}$ and set of germs $\mathcal{E}_{i-1}$. Moreover, for each $f \in \mathcal{E}_{i-1}$, we have given positive numbers $r_{1}(f), \ldots, r_{n+1}(f)$ and an analytic function $P_{i}^{\prime \prime}[f]$ defined on

$$
U_{i}^{i-1}(f)=\left\{\left(z_{i}^{i-1}, \ldots, z_{n}^{i-1}, u\right):\left|z_{i}^{i-1}\right|<r_{i}(f), \ldots,|u|<r_{n+1}(f)\right\}
$$

such that $u \backslash P_{i}^{\prime \prime}[f]$ (hence mult $\left.{ }^{\prime}\left(P_{i}^{\prime \prime}[f]\right)<\infty\right)$. Consider

$$
d_{i}:=\min \left\{\operatorname{mult}^{\prime}\left(P_{i}^{\prime \prime}[f]\right): f \in \mathcal{E}_{i-1}\right\} .
$$

Define $\left\{z_{i}, z_{i+1}^{i}, \ldots, z_{n}^{i}\right\}$ by the formulas $z_{i}^{i-1}=z_{i}$ and $z_{j}^{i-1}=z_{j}^{i}+\lambda_{j}^{i} z_{i}$, with $\lambda_{j}^{i}$ real for $j>i$. For any $f \in \mathcal{E}_{i-1}$, we express $P_{i}^{\prime \prime}[f]$ respect to the new variables $z_{1}, \ldots, z_{i}, z_{i+1}^{i}, \ldots, z_{n}^{i}, u$. Then, if the $\lambda_{j}^{i}$ 's are chosen generic enough, there exists $f \in \mathcal{E}_{i-1}$ such that wideg $_{z_{i}}\left(P_{i}^{\prime \prime}[f]\right)=d_{i}$. Define the non-empty set

$$
\mathcal{E}_{i}^{1}:=\left\{f \in \mathcal{E}_{i-1}: \operatorname{wideg}_{z_{i}}\left(P_{i}^{\prime \prime}[f]\right)=d_{i}\right\} .
$$

For each $f \in \mathcal{E}_{i}^{1}$ we can diminish the numbers $r_{i}(f), \ldots, r_{n+1}(f)$ so that the function $P_{i}^{\prime \prime}[f]$ can be decomposed over the open subset

$$
U_{1}^{i}(f):=\left\{\left(z_{i}, z_{i+1}^{i}, \ldots, z_{n}^{i}, u\right):\left|z_{i}\right| \leq r_{i}(f), \ldots,|u| \leq r_{n+1}(f)\right\}
$$

as $P_{i}^{\prime \prime}[f]=\phi_{i} P_{i}[f]$, where $\phi_{i}$ does not vanish anywhere in $U_{i}^{i}$ and $P_{i}[f]$ is a pseudopolynomial of the form

$$
P_{i}[f]\left(z_{i}, \ldots, u\right):=z_{i}^{d_{i}}+\sum_{j=1}^{d_{i}-1} z_{i}^{j} \alpha_{i}^{j}[f]\left(z_{i+1}^{i}, \ldots, u\right) .
$$

View $P_{i}[f]$ as a family of polynomials of $\mathbb{C}_{d_{i}}\left[z_{i}\right]$ parametrized by the open subset

$$
U_{2}^{i}(f):=\left\{\left(z_{i+1}^{i}, \ldots, z_{i+1}^{i}, u\right):\left|z_{i+1}^{1}\right| \leq r_{i+1}(f), \ldots,|u| \leq r_{n+1}(f)\right\} .
$$

By analogy with Stage 1 , we define numbers $m_{i}^{0}, s_{i}^{0}, k_{i}^{0}$, a stratum $S_{i}^{0} \in \mathbb{C}_{d_{i}}\left[z_{i}\right]$, and a decreasing sequence of subsets $\mathcal{E}_{i}^{1} \supset \mathcal{E}_{i}^{2} \supset \mathcal{E}_{i}^{3} \supset \mathcal{E}_{i}$. Moreover, for each function $f \in \mathcal{E}_{i}$ we construct functions $P_{i+1}^{\prime}[f]$ and $P_{i}^{\prime \prime}[f]$ analytic in $z_{i+1}^{i}, \ldots, u$, such that $P_{i+1}^{\prime}[f]=u^{k_{i}^{0}} P_{i}^{\prime \prime}[f]$ and $u \chi_{i}^{\prime \prime}[f]$.

Stage $\boldsymbol{n}+\mathbf{1}$. For any $f \in \mathcal{E}_{n}$, the function $P_{n}^{\prime \prime}[f]$ is a unit in $\mathbb{K}\{u\}$. Moreover, in the previous stage we have constructed a coordinate system $\left(z_{1}, \ldots, z_{n}\right)$ of $\mathbb{K}^{n}$ 
which will be said to be a good coordinate system with respect to $T$. We will define the subset

$$
\mathcal{E}_{T} \subset \pi_{n}^{-1}(T)
$$

of optimal germs of $\pi_{n}^{-1}(T)$ with respect to the coordinate system $\left(z_{1}, \ldots, z_{n}\right)$ as $\mathcal{E}_{T}:=\mathcal{E}_{n}$. This finishes the Search for Optimal Germs.

Proof of Proposition 9. We will start by proving that being optimal respect to a fixed good coordinate system $\left(z_{1}, \ldots, z_{n}\right)$ is a finitely determined property, i.e., that there exists $s>r$ with the following property: given any $x \in X$ and any $g \in \tilde{I}_{x} \cap \mathbf{m}_{x}^{s+1}$, a germ $f \in T_{x}$ belongs to $\mathcal{E}_{T}$ if and only if $f+g$ belongs to $\mathcal{E}_{T}$. By convenience of the reader we repeat the statement of Proposition 4.2 of [18]:

$(\dagger)$ For any two natural numbers $k$ and $p$ there exists a third one $L(k, p)$ such that for any $f \in \mathbb{C}\left\{z_{1}, \ldots, z_{n}\right\}$ with $\operatorname{wideg}_{z_{1}}(f)=k$ and $g \in \mathbf{m}^{L(k, p)}$ the following property holds: consider analytic functions $\phi, \phi^{\prime}, P, P^{\prime}$, such that $\phi(O) \neq 0$, $\phi^{\prime}(O) \neq 0$ and $f=P \phi, f+g=P^{\prime} \phi^{\prime}$ in some neighbourhood of $O$, where $P$ and $P^{\prime}$ are Weierstrass polynomials in $z_{1}$ of degree $k$. Then $\phi-\phi^{\prime}$ and $P-P^{\prime}$ are in $\mathbf{m}^{p}$.

In order to choose $s$ we define the following numbers recursively:

$$
\begin{gathered}
a_{n}:=k_{n-1}^{0}+\max \left\{d_{n}+1, L\left(d_{n}, d_{n}+k_{n}^{0}\right)\right\}, \\
a_{i}:=k_{i-1}^{0}+\max \left\{d_{i}+1, L\left(d_{i}, a_{i+1}+d_{i}\right)\right\} \quad \text { for } \quad 2 \leq i \leq n-1, \\
a_{1}:=\max \left\{d_{1}+1, L\left(d_{1}, a_{2}+d_{1}\right)\right\} .
\end{gathered}
$$

Fix $s:=a_{1}$. With this choice, taking into account $(\dagger)$ along the procedure of Search for Optimal Germs, it is easy to show that given $f \in T_{x}$ and $g \in \tilde{I}_{x} \cap \mathbf{m}_{x}^{s+1}$ then $f \in \mathcal{E}_{T}$, if and only if $f+g \in \mathcal{E}_{T}$.

Now we suppose that $f \in \mathcal{E}_{T}$. We will show that $f$ and $f+g$ are topologically equivalent. Consider a coordinate function $y$ for the affine line $\mathbb{A}_{\mathbb{K}}^{1}$, and define the $\mathbb{A}_{\mathbb{K}}^{1}$-family $F:=f+y g$. The germ $F_{\mid y}$ is optimal respect to $\left(z_{1}, \ldots, z_{n}\right)$ for any $y \in \mathbb{A}_{\mathbb{K}}^{1}$ for being $y g \in \tilde{I}_{x} \cap \mathbf{m}_{x}^{s+1}$. It is easy to check that the algorithm can be applied to the family $F$, taking as initial coordinate system $\left(z_{1}, \ldots, z_{n}\right)$ and having in each step the trivial coordinate change. Denote by $A$ the subset of $\mathbb{A}_{\mathbb{K}}^{1}$ constructed in the algorithm. The Search for Optimal Germs has been designed in a compatible way with the algorithm so that $F_{\mid y}$ is optimal with respect to $\left(z_{1}, \ldots, z_{n}\right)$ if and only if $y \in \mathbb{A}_{\mathbb{K}}^{1} \backslash A$. Therefore, $A=\emptyset$. Applying Proposition 16 we obtain the topological equivalence of $f=F_{\mid 0}$ and $f+g=F_{\mid 1}$.

We are ready to prove the statement of the proposition. Recall that $\tilde{I}$ is generated over $W$ by the functions $f_{1}, \ldots, f_{m}$. Given any $x \in W$, we denote by $f_{i, x}$ the Taylor expansion of $f_{i}$ at $x$. Consider the $\mathbb{K}$-vector space $E$ of polynomials of degree bounded by $S$, let $\left\{g_{1}, \ldots, g_{N}\right\}$ be a basis of $V$. The product $W \times V^{m}$ is an open subset of $\mathbb{K}^{n+m N}$, and a point of it is represented by a $m N+1$ uple $\left(x, \lambda_{1}^{1}, \ldots, \lambda_{N}^{1}, \ldots, \lambda_{1}^{m}, \ldots, \lambda_{m}^{N}\right)$, with $x \in W$ and $\lambda_{j}^{i} \in \mathbb{K}$. Consider the 
$W \times V^{m}$-family defined by the unique analytic function $F$ in a neighbourhood of $\{O\} \times W \times V^{m}$ in $\mathbb{K}^{n} \times W \times V^{m}$ satisfying

$$
F_{\mid\left(x, h_{j}^{i}\right)}=\sum_{i=1}^{m} \sum_{j=1}^{N} g_{j} f_{i, x}
$$

for any $\left(x, h_{j}^{i}\right) \in W \times V^{m}$. Associated with $F$, we have a natural analytic mapping

$$
\psi: W \times V^{m} \rightarrow J^{s}(W, \mathbb{K})
$$

which assigns to $\left(x, h_{j}^{i}\right)$ the $s$-jet of the germ $F_{\mid\left(x, h_{j}^{i}\right)}$ viewed as a germ at $x$. As $T$ is a $r$-determined $(C)$-analytic subset of $J^{\infty}(X, \tilde{I})$ and $s>r$, the subset $T^{\prime}:=\psi_{s}^{-1}\left(\pi_{s}^{\infty}(T)\right)$ is a closed $(C)$-analytic subset of $(W \backslash \partial X) \times V^{m}$. Therefore, we can consider the $T^{\prime}$-family of functions obtained by restriction of $F$.

It is easy to check that $\psi\left(T^{\prime}\right)=\pi_{s}^{\infty}(T)$. Therefore, there exists $t^{\prime} \in T^{\prime}$ such that $\psi\left(t^{\prime}\right)$ is the $s$-jet of an optimal germ of $T$ with respect to the coordinate system $\left(z_{1}, \ldots, z_{n}\right)$. Taking into account the way we have designed the algorithm and the Search for Optimal Germs, and the fact that being optimal with respect to the fixed coordinate system is an $s$-determined property, it follows easily that

(1) the algorithm can be applied to the $T^{\prime}$-family of functions $F$ choosing at each stage the trivial coordinate change;

(2) a point $t^{\prime} \in T^{\prime}$ belongs to the $(C)$-analytic proper subset $A^{\prime} \subset T^{\prime}$ constructed in the algorithm if and only if $\psi\left(t^{\prime}\right)$ is not the $s$-jet of an optimal germ of $T$ with respect to the fixed coordinate system.

Therefore, $A^{\prime}$ is of the form $\psi^{-1}\left(A^{\prime \prime}\right)$ with $A^{\prime \prime} \subset J^{s}(X, \tilde{I})$. The fact that $A^{\prime \prime}$ is $(C)$-analytic is easily deduced from the facts that $\psi_{\mid X \times V^{m}}: X \times V^{m} \rightarrow J^{s}(X, \tilde{I})$ is an epimorphism of trivial analytic vector bundles, and that $A^{\prime}$ is $(C)$-analytic. Define $A$ as the $s$ determined $(C)$-analytic subset $A:=\left(\pi_{s}^{\infty}\right)^{-1}\left(A^{\prime \prime}\right)$.

Consider $f, f^{\prime}$ in the same connected component of $T \backslash A$. Then there exist $t, t^{\prime}$ in the same connected component of $T^{\prime} \backslash A^{\prime}$ such that $\psi(t)=\pi_{s}^{\infty}(f)$ and $\psi\left(t^{\prime}\right)=\pi_{s}^{\infty}\left(f^{\prime}\right)$. By Proposition 16 we have that $F_{\mid t}$ and $F_{\mid t^{\prime}}$ are topologically equivalent. For being $t, t^{\prime} \notin A$, the germs $F_{\mid t}$ and $F_{\mid t^{\prime}}$ are optimal with respect with the coordinate system $\left(z_{1}, \ldots, z_{n}\right)$. As $f$ and $f^{\prime}$ have respectively the same $s$-jet that $F_{\mid t}$ and $F_{\mid t^{\prime}}$, and optimal germs are topologically $s$-determined, we conclude the topological equivalence of $f$ and $f^{\prime}$.

\section{References}

[1] D. V. Anosov, S. Kh. Aranson, V. I. Arnold, I. U. Bronshtein, V. Z. Grines and Yu. S. Il'yashenko, Ordinary Differential Equations and Smooth Dynamical Systems, Springer, 1997.

[2] E. Bierstone and P. D. Milman, Relations among aanlytic functions I, Ann. Inst. Fourier, Grenoble 37, no. 1 (1987), 187-239.

[3] F. Bruhat and H. Whitney, Quelques propietés fondamentel des ensembles analytiques-réels, Comentarii Mathematici Helvetici 33, no. 1 (1959), 132-160. 
[4] H. Cartan, Variétés analytiques réelles et variétés analytiques complexes, Bull. Soc. Math. France 85 (1957), 77-79.

[5] J. Fernández de Bobadilla, Generic approximations of non-isolated singularities of finite codimension with respect to an i.c.i.s, Bull. London Math. Soc. 35 no. 6 (2003), 812-816.

[6] J. Fernández de Bobadilla, Relative Morsification Theory, Topology 43 no. 4 (2004), 925982.

[7] E. Looijenga, Structural stability of families of $C^{\infty}$ functions and the canonical stratification of $C^{\infty}(N)$, Thesis, Amsterdam University. Preprint I.H.E.S., 1974.

[8] T. de Jong, Some classes of line singularities, Math. Zeitschr. 198 (1998), 493-517.

[9] R. Pellikaan, Finite determinancy of functions with non-isolated singularities, Proc. London Math. Soc. (3) 57 (1998), 357-382.

[10] R. Pellikaan, Deformations of hypersurfaces with a one dimensional singular locus, J. Pure. Appl. Algebra 67, no. 1 (1990), 49-71.

[11] A. du Plessis, On the genericity of topologically finitely-determined map-germs, Topology 21, no. 2, 131-156.

[12] R. Narashiman, Introduction to the Theory of Analytic Spaces, Lecture Notes in Mathematics 25, Springer, 1966.

[13] A. Nemethi, Hypersurface singularities with 2-dimensional critical locus, Proc. London Math. Soc. (2) 59 (1999), 922-938.

[14] D. Siersma, Isolated line singularities, in: Singularities (ed. P. Orlik), Proc. Symp pure math. 40:2, (Amer. Math. Soc., 1983), 485-496.

[15] D. Siersma, Singularities with critical locus a complete intersection and transversal type $A_{1}$, Topology and its Appl. 27 (1987), 51-73.

[16] E. H. Spanier, Algebraic Topology, McGraw-Hill, 1966.

[17] R. Thom, Local topological properties of differentiable mappings, in: Differential Analysis (Bombay Colloq., 1964) Oxford Univ. Press, London, 1964, 191-202.

[18] A. N. Varchenko, Local topological properties of analytic mappings, Math. USSR Izvestija 7, no. 4 (1973), 883-917.

[19] A. N. Varchenko, Local topological properties of differentiable mappings, Math. USSR Izvestija 8 (1974), 1033-1082.

[20] A. Zaharia, Topological properties of certain singularities with critical locus a 2-dimensional complete intersection, Topology and its Appl. 60 (1994), 153-171.

J. Fernández de Bobadilla

Mathematisch Instituut

Universiteit Utrecht

Postbus 80010

3508TA Utrecht

The Netherlands

e-mail: bobadilla@math.uu.nl

(Received: October 30, 2002)

\section{(17) To access this journal online: \\ (If) http://www.birkhauser.ch}

